\title{
Characterization of surface motion patterns in highly deformable soft tissue organs from dynamic MRI: An application to assess 4D bladder motion
}

\author{
Karim Makki ${ }^{a, *}$, Amine Bohi $^{a}$, Augustin C. Ogier ${ }^{a}$, and Marc Emmanuel Bellemare ${ }^{a}$ \\ ${ }^{a}$ Aix Marseille Univ, Université de Toulon, CNRS, LIS, Marseille, France
}

\begin{abstract}
Background and objectives: Dynamic Magnetic Resonance Imaging (MRI) may capture temporal anatomical changes in soft tissue organs with high-contrast but the obtained sequences usually suffer from limited volume coverage which makes the high-resolution reconstruction of organ shape trajectories a major challenge in temporal studies. Because of the variability of abdominal organ shapes across time and subjects, the objective of the present study is to go towards 3D dense velocity measurements to fully cover the entire surface and to extract meaningful features characterizing the observed organ deformations and enabling clinical action or decision.
\end{abstract}

Methods: We present a pipeline for characterization of bladder surface dynamics during deep respiratory movements. For a compact shape representation, the reconstructed temporal volumes were first used to establish subject-specific dynamical 4D mesh sequences using the large deformation diffeomorphic metric mapping (LDDMM) framework. Then, we performed a statistical characterization of organ dynamics from mechanical parameters such as mesh elongations and distortions. Since we refer to organs as non-flat surfaces, we have also used the mean curvature changes as metric to quantify surface evolution. However, the numerical computation of curvature is strongly dependant on the surface parameterization (i.e. the mesh resolution). To cope with this dependency, we employed a non-parametric method for surface deformation analysis. Independent of parameterization and minimizing the length of the geodesic curves, it stretches smoothly the surface curves towards a sphere by minimizing a Dirichlet energy. An Eulerian PDE approach is used to derive a shape descriptor from the curve-shortening flow. Intercorrelations between individuals' motion patterns are computed using the Laplace-Beltrami Operator (LBO) eigenfunctions for spherical mapping.

Results: Application to extracting characterization correlation curves for locally-controlled simulated shape trajectories demonstrates the stability of the proposed shape descriptor. Its usability was shown on MRI acquired for seven healthy participants for which the bladder was highly deformed by maximum of inspiration. As expected, the study showed that deformations occured essentially on the top lateral regions. 
Conclusion: Promising results were obtained, showing the organ in its 3D complexity during deformation due to strain conditions. Smooth genus-0 manifold reconstruction from sparse dynamic MRI data is employed to perform a statistical shape analysis for the determination of bladder deformation.

Keywords: Pelvic floor, motion estimation, dynamic MRI, statistical shape analysis, geodesic distances, differential geometry

\section{Introduction}

Pelvic floor disorders affect approximately $50 \%$ of women older than 50 years [1]. Related health problems such as urinary and fecal incontinences get worse with age which affects activities of daily living. Dynamic MRI examinations are now essential for the investigation of the pelvic area as they allow a non-invasive observation of the main organ deformations [2]. Current clinical practice involves 2D dynamic MRI acquiring a single sagittal plane per time frame [3]. However, in-plane and out-of-plane deformations can simultaneously occur, thus limiting the ability to assess the effective 3D deformations from the $2 \mathrm{D}$ images. In the context of pelvic floor, 3D imaging has remained exclusive to static MRI so far, in order to observe pelvic floor anatomy at rest during instructed apnea. In fact, the MRI-modalities are still too slow to follow the movements within a reasonably short duration. Moreover, most of non-invasive and fast 3D acquisitions suffer from intrinsically low volume rates $[4,5]$.

Assuming that $58 \%$ of women who undergo surgery have reported a recurrence of pelvic disorders [6], one of the most challenging tasks is to enhance the understanding of the organ connections and their morphological changes under strain conditions. Beyond visual inspection by a radiologist, a more relevant quantitative information about pelvic organ deformations is required to support surgeons' needs for optimization of implant position and post-surgical follow-up of patients. To address these needs, some recent studies [7, 8] have begun to create a biomechanical model of organ interactions, while other studies focused on the role of $3 \mathrm{D}$ reconstruction in the assessment of organ motion [9, 10]. Both these approaches pave the way for a better understanding of organ behaviors and they would profit from an efficient descriptor of organ temporal 3D deformations. Indeed, such a tool could be appreciable to simplify the visualization and the statistical identification of the most common deformations, and to compare organ dynamics simulations to organ dynamics observations, to name a few gains. Furthermore, comparing organ movement between patients would allow to identify and grade abnormal motion in order to classify patients into healthy and pathological, or into subgroups sharing similar organ motion characteristics within large data sets, which will optimize surgical and non-surgical treatments by avoiding unnecessary duplication of effort across patients.

From a methodological point of view, in vivo characterization of the dynamical behavior of human joints, organs, and soft tissues during daily physical activities remains challenging because of the complexity and the non-linearity of their shape dynamics $[11,12,13,14,15$, 16]. During the last decade, statistical shape analysis tools have been shown to be clinically useful, in particular, to help understand patterns in large clinical data sets and to characterize 
the functioning soft tissue organs or anatomical structures undergoing large deformations [17, 18, 19, 20, 21, 22]. However, one should keep in mind that simply encoding deformations with some displacement vector fields is not sufficient to identify clinically relevant patterns of the induced organ deformations because of the complexity of their dynamics. Therefore, spacetime statistics should be based on robust and stable surface features or even better, geometric shape descriptors $^{1}$. In other words, the temporal feature changes should characterize the local shape deformations. Since shape space is not necessarily flat, then statistical tools derived from Euclidean geometry are not well adapted to study organ shape dynamics. Under such circumstances, it is practically meaningful to employ Riemannian geometry for generating shape trajectories which belong to non-linear manifolds [23, 24]. Relying on the notion of geodesic distances on manifolds to compute the shortest paths between two points on a curved surface [25], these tools cover topics that start from the fundamentals of general relativity theory $[26]$.

In practice, several studies for characterizing spatio-temporal shape trajectories have used longitudinal datasets aiming at quantifying: the shape growth over a long period of time [27] (intrasubject variations in geometry), or the shape variability in an inter-subject context (e.g. for atlas building at the population scale [28]). Longitudinal data consist of a collection of stationary scans (e.g. conventional high-resolution MRI scans) portraying anatomical changes during a large period of time (over weeks, months, or even years depending on the studied pathology or phenomenon). Such studies have served to highlight the need for clinicians to understand anatomical changes occurring during development or disease progression (e.g. linear geodesic regression model for shape time-series with sparse parameters [21, 19]). In the context of spatio-temporal bladder imaging [29], longitudinal samples are often limited by bladder volume variation which may cause an increase in scaling effects. A solution is to perform short-time non invasive imaging in order to assess the effective deformations of constant bladder volumes. The latter condition is essential to direct the radiotherapy of pelvic tumors with precision [30].

As compared to longitudinal studies, temporal studies using dynamic MRI may noninvasively capture anatomical changes during motion over a short period of time, but at the cost of having: (1) less spatio-temporal resolution of anatomical data sequences, (2) higher sensitivity to motion artifacts, and (3) an increased effect of image noise. Overcoming these difficulties that appear in sparse spatio-temporal data is a whole new challenge, especially when it becomes necessary to have a high spatio-temporal resolution of shape trajectory in order to carry out statistically significant studies.

In this paper, we have employed diffeomorphic statistical shape tools to evaluate organ surface dynamics. We show the strength of the approach by characterizing 3D MRI observations of bladder under strain conditions. Starting from sets of high resolution reconstructed temporal bladder volumes, we have first employed the large deformation diffeomorphic metric mapping (LDDMM) framework to encode the organ's large deformations with a reduced number of significant surface points while covering properties in shape geometry. A dy-

\footnotetext{
${ }^{1}$ The two terms are used interchangeably throughout the paper.
} 
namical quadrilateral mesh for the organ surface is then established to address the need for preserving neighborhood structures when computing geometric descriptors, that are classically used in biomechanics, such as mesh elongations and distortions. Then, characterizations of organ shape dynamics are performed by computing mean curvature changes. Moreover, a new geometric feature is also proposed. It allows detecting salient motion patterns from geodesic shortest length paths for mapping a shape to a sphere. It satisfies the "invariance" conditions of Kendall's shape space [31], by filtering out location, size and rotation. This feature may capture local surface variations, with no need to compute Riemann's tensor. This last point is an interesting advantage as computing tensors not only incurs high computational costs but also impacts numerical stability [32]. Results demonstrate that the proposed feature, possessing a high repeatability score of measures throughout cyclic shape trajectories, is much more numerically stable.

Since we are interested in comparing organ shapes in terms of their geometry rather than their size, we performed all comparisons in a common shape space. Indeed, motion patterns derived from the different feature vectors were projected onto the unit sphere $\mathbb{S}^{2}$ (point-topoint anatomical correspondences between these geometric features were established across subjects).

\section{Related work}

In the literature, some studies show attempts to quantify or model human soft tissue in a non-invasive manner during daily living activities [33, 18, 16]. In [34], the authors proposed a semi-automatic segmentation framework to track the motion of the tongue and to measure its internal deformation during speech and swallowing using dynamic MRI. In contrast, the temporal resolution was limited and the tongue trajectory was only represented by 26 reconstructed volumes, each with a voxel size of $1.875 \times 1.875 \times 1.875 \mathrm{~mm}$. In [35, 16], a first attempt to quantify the ankle joint motion patterns through a combination of static and dynamic MRI data was presented. A tracking of bones and surrounding soft tissues was performed by estimating a dense deformation field covering the entire field of view from the static scan to dynamic time frames using the Log Euclidean Polyrigid registration Framework (LEPF) [36]. However, in the context of pelvic floor dynamics, most biomechanics simulation experiments have been hampered by the lack of relevant data due to limitations of spatiotemporal resolution of dynamic MRI [7, 8]. In [8], 2D dynamic MRI images were combined with 3D biomechanical models in order to extrapolate the complete 3D dynamic motion of abdominal organs. A validation attempt was performed by checking that the reconstructions were well conducted from the first scan towards the end of dynamic sequence. However, the validation itself was not founded on a clinically relevant ground truth (i.e. the validation was not performed in the high resolution domain). Furthermore, only two simulated sequences have been used to validate the model.

The above issues were addressed in [9]: a high-resolution spatio-temporal reconstruction of the non-linear dynamics of an abdominal organ motion was introduced for designing a continuous-time dynamics that allowed us to infer inter-frame deformations. Promising results were obtained, showing the bladder in its 3D complexity during deformation due to 
strain conditions with an estimation of the most deformed tissue areas. However, bladder motions were quantified based on the temporal changes in Jacobian determinant of the estimated deformation fields. Although this parameter can quantify the organ volume changes, it fails to identify its local morphological changes throughout motion. Since we are interested in comparing differences in geometry (rather than in size), these high-resolution temporal data were then employed to introduce a compact characterization of moving bladder surfaces through the use of a geodesic-based shape descriptor in [10]. In this paper, we have extended the study group to include more subjects and we have proposed a technique to simulate pathological data sequence. Moreover, more results for comparing between shape descriptors and individual motion patterns were provided to show how the use of such methods is intended to ultimately inform clinical practice and research.

In [37], a characterization of pelvic organ dynamics was proposed using diffeomorphic registration on dynamic $2 \mathrm{D}$ slices. The objective of the present study is to go towards 3D dense velocity measurements to fully cover the entire surface. Furthermore, most of the descriptors used in [37] were based on Euclidean geometry (i.e. the geometry of a flat space). Proposing new shape descriptors which generalize Euclidean geometry to non-flat or curved spaces eventually becomes necessary with the increase in the number and complexity of motion patterns to be recognized.

\section{Methods}

\subsection{Dynamic quadrilateral mesh}

\subsubsection{Surface parameterization}

In a first step, we have extracted an initial control mesh from the reconstructed bladder volumes (i.e. 3D binary masks obtained using the methods introduced in [9]) at the first time frame using the marching cubes algorithm [38]. The simplicial surface is then converted to a quasi-regular quadrilateral mesh $(K, \mathcal{M})$ such that $\mathcal{M}=\left\{x_{1}, x_{2}, \ldots x_{n}\right\}, x_{i} \in \mathbb{R}^{3}$ is the set of vertex positions, and $K$ is a cubical complex determining the topological type of the mesh by specifying the connectivity of vertices, faces, and edges. To fully cover the entire surface with an extremely low number of meaningful variables, we have used a robust algorithm presented in [39], providing a uniform distribution of vertices over the manifold by iteratively refining the initial control mesh until a pure quad mesh is obtained. This algorithm avoids irregularity

problems at the shape poles, such as vertex singularities encountered in [7]. Fig. 1 illustrates the quality of an obtained quadrilateral mesh.

\subsubsection{Estimation of smooth vertex trajectories}

In a second step, we propose to track the mesh vertices during respiratory motion while preserving their connectivities. This allows for constructing a spatio-temporal structured meshes which might also be used for deriving some biomechanical properties of the organ dynamics such as strains and stresses using finite element methods which are required for establishing biomechanical models (i.e. pure quad meshes are often desired in CAD applications). 

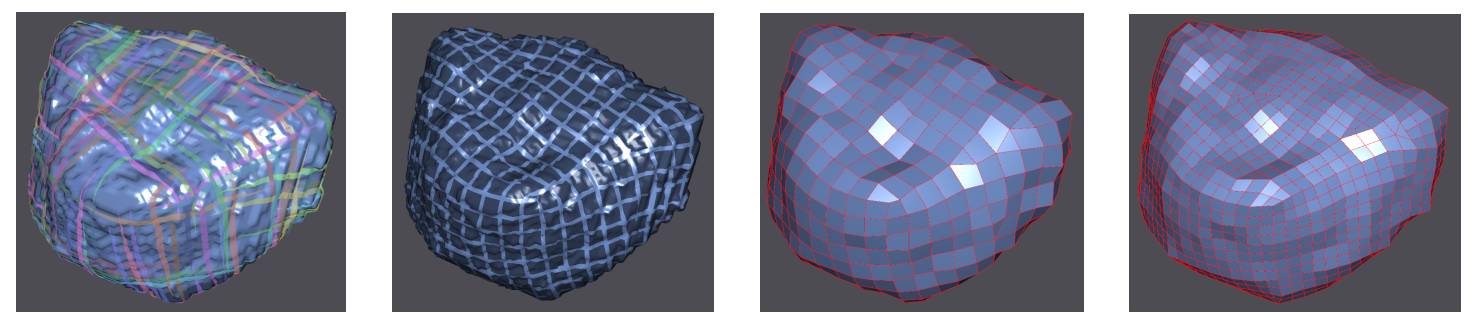

Figure 1: Parameterization of the bladder surface, from left to right: the orientation field, the position field, the quad dominant mesh, and the resulting pure quad mesh.

Our model for shape analysis focus on deformations represented by diffeomorphisms acting on landmarks. To determine point correspondences, a mesh-to-volume registration is performed. In other words, we propose to track the set of mesh vertices using the LDDMM framework that has been heuristically shown to produce natural deformation paths in the space of diffeomorphisms without having to use point-to-point correspondences between source and target point sets [40]. A smooth and continuous-time trajectory of each vertex is estimated throughout the organ range of motion (vertices travel along geodesic curves).

The principle of control-points-based LDDMM for estimating a diffeomorphic mapping is as follows:

Given a set of $N$ control points $\left\{q_{i}\right\}_{i \in 1, \ldots, N}$, and a set of $N$ corresponding momentum vectors of $\mathbb{R}^{3}\left\{\mu_{i}\right\}_{i \in 1, \ldots, N}$, the velocity vector in the tangent space $\mathcal{T}_{x} \mathcal{M}$ of the parametric surface $\mathcal{M}$ at a point $x \in \mathcal{M}$, is obtained through the use of a Gaussian convolution filter:

$$
v: x \in \mathcal{M} \mapsto v(x)=\sum_{i=1}^{N} K\left(x, q_{i}\right) \cdot \mu_{i}
$$

where $K\left(x_{i}, x_{j}\right)=\exp \left(-\left\|x_{i}-x_{j}\right\|^{2} / \sigma^{2}\right)$ is a Gaussian kernel to ensure smooth geodesic shooting.

The temporal evolution of the organ velocity vector field can be modeled by the following Hamilton's equations of motion:

$$
\left\{\begin{array}{l}
\dot{q}(s)=K(q(s), q(s)) \cdot \mu(s) \\
\dot{\mu}(t)=-\frac{1}{2} \nabla_{q}\left\{K(q(s), q(s)) \cdot \mu(s)^{\top} \mu(s)\right\}
\end{array}\right.
$$

The solutions to this Hamiltonian are then the same as the geodesics on a Riemannian manifold. The numerical integration of these PDEs, performed using a second-order RungeKutta scheme, gives a flow of a time-dependent velocity vector field parameterized with $q(s)$ and $\mu(s)$ :

$$
v: x \in \mathbb{R}^{3} \times s \in[0,1] \mapsto v(x, s)=\sum_{i=1}^{N} K\left(x, q_{i}(s)\right) . \mu_{i}(s)
$$

The temporal displacement of each tracked point $x \in \mathcal{M}$ is governed by the following autonomous first order ODE: 


$$
\dot{x}(s)=v(x(s), s) \quad \text { subject } \quad \text { to } \quad x(0)=x
$$

Finally, the solution of this ODE yields a flow of diffeomorphisms starting from the source points (i.e. starting from the identity in the space of transformations), $\Phi_{q, \mu}(., s)$ : $\mathbb{R}^{3} \times[0,1] \mapsto \mathbb{R}^{3}$, such that $\Phi_{q, \mu}(., 1)=I d+\int_{0}^{1} v\left(\Phi_{q, \mu}(., s)\right) d s$ is the end-point of the geodesic flow matching the given point sets.

The overall algorithm for vertex tracking is described in Algorithm 1, with the following notations: $L$ is the length of the dynamic sequence, $\mathcal{M}_{t}$ gives the locations of mesh vertices at time $t, \mathcal{C}_{t}$ is the entire $3 \mathrm{D}$ surface point cloud at time $t\left(\mathcal{M}_{t}\right.$ is a proper subset of $\left.\mathcal{C}_{t}\right)$. Note that the registration problem is solved by iteratively minimizing the following loss function:

$$
f(q, \mu)=d\left(\mathcal{C}_{t+1}, \Phi_{q, \mu}\left(\mathcal{M}_{t}\right)\right)+R(q, \mu)
$$

where the first term measures data-attachment while the second regularization term represents the norm of the deformation.

Algorithm 1 LDDMM-based 4D quad mesh generation

- Inputs: - Initial mesh structure $\left(K, \mathcal{M}_{0}\right)$.

- Reconstructed segmentation contours $\mathcal{C}_{t}, \quad t \in\{1 \ldots L-1\}$

- Motion estimation: Estimate forward successive vertex trajectories using the LD$\operatorname{DMM}\left\{\mathcal{M}_{t+1}\right\}_{t=0, \ldots, L-1}$ such that $\mathcal{M}_{t} \subset \mathcal{C}_{t}$, by aligning $\mathcal{M}_{t}$ and $\mathcal{C}_{t+1}$ :

- for $t$ in $0 \ldots L-1$ :

1. Initialize $\left\{q_{i}^{t}(s)\right\}_{i \in 1, \ldots, N}$, and $\left\{\mu_{i}^{t}(s)\right\}_{i \in 1, \ldots, N}$.

2. Compute velocities $v^{t}$ according to Eq 3 .

3. Integrate the flow $\Phi_{q, \mu}^{t}(x, s)=I d_{\mathbb{R}^{3}}+\int_{0}^{1} v^{t}\left(\Phi_{q, \mu}(x, s)\right) d s, \quad \forall x \in \mathcal{M}_{t}$, by minimizing the cost function defined in Eq 5 .

- Output: $4 \mathrm{D}$ quad mesh sequence $\left(K, \mathcal{M}_{t}\right)_{t=0,1, \ldots, L-1}$.

To validate the tracking process, we propose to compute the following error:

$$
E=\frac{1}{n} \sum_{p=1}^{n} \operatorname{dist}\left(x_{p}, \mathcal{C}_{L-1}\right)
$$

where: $n$ is the total number of tracked vertices, $x_{p} \in \mathcal{M}_{L-1}$, and $\operatorname{dist}\left(x_{p}, \mathcal{C}_{L-1}\right)$ is the Euclidian $\ell^{2}$ distance between $x_{p}$ and the closest point $x_{l}$ in the last reconstructed surface $\mathcal{C}_{L-1}$. A propagated mean error of $0.63 \pm 0.06 \mathrm{~mm}$ was obtained across all subjects. The resulting error was always inferior to $1 \mathrm{~mm}$ which reflects the tracking accuracy level for a 
given isotropic voxel size of $1 \times 1 \times 1 \mathrm{~mm}$. Fig. 2 shows the quality of our $3 \mathrm{D}+t$ quadrilateral mesh reconstruction based on smoothly tracking vertices using the LDDMM while keeping connectivity unchanged.

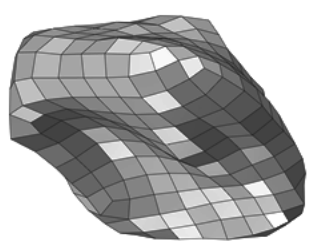

$$
t=0
$$

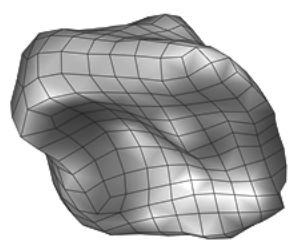

$t=55$

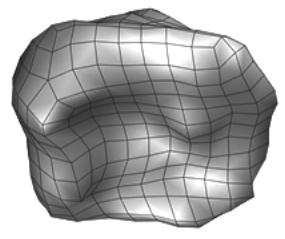

$t=152$

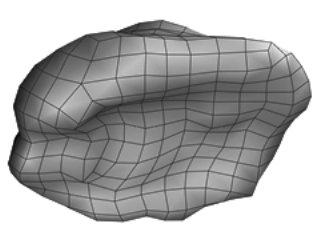

$t=185$

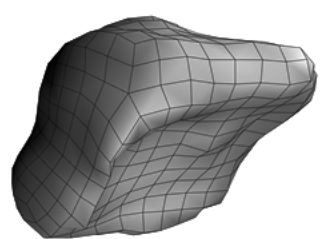

$t=230$

Figure 2: Example of 4D reconstructed quad mesh for the bladder during forced respiratory motion.

\subsection{Shape descriptors}

\subsubsection{Mesh elongation and distortion}

Shape elongations and distortions have been used in medical imaging, in particular to characterize the deformations of pelvic organs in the plane from dynamical shape contour points [37]. However, such characterizations are prone to biased interpretation of organ motion patterns because of the out-of-plane problem. To overcome these limitations, we propose a 3D extension method of these 2D feature maps, compatible with the reconstructed quad meshes.

\section{Mesh elongation:}

The mesh elongation, equivalent to the Green-Lagrange deformation descriptor, is a commonly used feature in biomechanics for characterizing local mesh deformations based on the spacing changes in the neighborhood of each vertex. Consider a vertex $x_{i}^{j}(t)$ and its four nearest neighbors $\left\{x_{i+1}^{j}(t), x_{i-1}^{j}(t), x_{i}^{j+1}(t), x_{i}^{j-1}(t)\right\}$ belonging to the mesh at the $t^{\text {th }}$ time frame, and a vertex $x_{i}^{j}(t+1)$ and its neighbors $\left\{x_{i+1}^{j}(t+1), x_{i-1}^{j}(t+1), x_{i}^{j+1}(t+1), x_{i}^{j-1}(t+1)\right\}$, their homologous vertices at the $(t+1)^{t h}$ time frame (where $i$ and $j$ are used for indexing mesh local connectivities). We compute $\hat{d}(t)$ and $\hat{d}(t+1)$, the average Euclidean distance between $x_{i}^{j}(t)$ and its neighbors (resp. between $x_{i}^{j}(t+1)$ and its neighbors). Then, the elongation measure is given by:

$$
E(k)=\frac{\hat{d}(t+1)-\hat{d}(t)}{2 \hat{d}(t)}
$$

If $E(t)=0$, then no deformation has occurred, else if $E(t)>0$, the neighborhood of the vertex has expanded. Otherwise, the neighborhood has shrunk. 


\section{Mesh distortion:}

The dihedral angles are defined as the angles between the two normal vectors to each two adjacent faces [41]. And the temporal change in these angles during motion is considered as the temporal distortion of the quad mesh.

For two adjacent quad faces $F_{1}=\left\{x_{1}, x_{2}, x_{3}, x_{4}\right\}$ and $F_{2}=\left\{x_{1}, x_{2}, x_{3}^{\prime}, x_{4}^{\prime}\right\}$, with normals $N_{1}$ and $N_{2}$, respectively, the local dihedral angle is computed as follows:

$$
\theta=\arccos \left(\frac{N_{1} \cdot N_{2}}{\left\|N_{1}\right\| \times\left\|N_{2}\right\|}\right)
$$

Assuming that the adjacent faces to each vertex of the initial quad mesh (at $t=0$ ) were maintained the same throughout the sequence, then both vertices $x_{i}^{j}(t)$ and $x_{i}^{j}(t+1)$ have always the same adjacent faces that we note $\left\{F_{1}, F_{2}, F_{3}, F_{4}\right\}$. If we consider $\theta(t)$ and $\theta(t+1)$ as the maximal dihedral angles among the six possible couple combinations of adjacent faces at times $t$ and $t+1$, respectively, then, the temporal mesh distortion around $x_{i}^{j}$ is determined by:

$$
D(t)=|\theta(t+1)-\theta(t)|
$$

If $D(t)=0$, then no local deformation has occurred. Otherwise, the neighborhood has distorted.

\subsubsection{A novel geodesic-based feature for characterization of organ surfaces:}

In the field of computer graphics and computational geometry, shortest path problem on both flat and curved domains is a well-known problem. Several techniques have been proposed for estimating shortest path lengths, also known as geodesic distances, on curved domains. One of the most popular computational methods is the Dijkstra algorithm for finding the shortest paths between nodes in a weighted graph [42]. However, this algorithm computes just a rough approximation of the true distances. Its major drawback is that the direction along which distance increases is partially ignored since only horizontal and vertical displacements are allowed on the grid. Then, it becomes clear, for instance, that this method will overestimate the straight-line Euclidean distance of any diagonal path crossing a regular grid. To circumvent this problem, Sethian introduced the fast marching algorithm which is a far reaching generalization of the Dijkstra algorithm [43]. The fast marching algorithm computes the geodesic distance (in the viscosity sense) in $O(n . \log (n))$ operation. Equivalently it solves the following boundary value problem (BVP) of the Eikonal PDE by front propagation:

$$
\left\{\begin{array}{l}
|\nabla L(x)|=1, \quad x \in \Omega, \\
L(\partial \Omega)=0,
\end{array}\right.
$$


where $\Omega$ is an open set of $\mathbb{R}^{n}$ with well-behaved boundary, $n=\operatorname{card}(\Omega)$, and $L$ stands for the distance or length. However, being nonlinear (hyperbolic), the Eikonal PDE is just simple to state but not at all easy to solve, in particular for two-point BVPs which will be the case in the present work. Subsequently, Crane et al. proposed the heat method for geodesic distance computation [44]. In the heat method, the shortest path problem is reformulated in a more elegant way by transforming it into two simpler linear elliptic problems, thus allowing the main problem to be solved in three steps:

- Integrate the heat flow for a source point across the grid/mesh (problem 1).

- Compute the normalized gradient for the heat (a simple change of variable).

- Recover the true distance from the normalized gradient by solving a Poisson equation (problem 2).

In this paper, we first propose to point out the relation between the heat method and an Eulerian PDE approach, that has been proposed in [45] for estimating tissue thickness between two non-intersecting boundaries, which in its turn, decomposes the shortest path problem into two elliptic problems using the same change of variable in-betweens. Then, we combine them to derive a robust shape descriptor which is purely based on geodesic distances and which will enable us to quantify the non-linear deformations of organ surface with respect to the sphere. The latter technique has been tremendously used for tissue thickness estimation [45, 46, 47], while, surprisingly enough, it has been used only for this particular problem, regardless of its potential to provide accurate geodesic distance maps in a more general setting since it can also handle multiple segment domains with appropriate boundary conditions. In short, the present work describes its potential to estimate the geodesic distance maps related to the mapping of a smooth surface into a sphere. In the following, we will explain in more detail how geodesic distance maps are estimated and then employed to derive our shape descriptor.

\section{Heat flow integration:}

First of all, let us introduce the heat transfer problem and emphasize the effect of the imposed boundary conditions on the solution. Consider the basic form of the heat equation:

$$
\frac{\partial h(x, t)}{\partial t}=\Delta h(x, t)
$$

Consider the 1-point BVP:

$$
\frac{\partial h(x, t)}{\partial t}=\Delta h(x, t) \quad \text { s.t } \quad h(\partial \Omega)=1
$$

The time-dependant solution of the above stated BVP is used in [48] to approximate the heat diffusion from a single source $\partial \Omega$ on a smooth manifold to all other points of the manifold. In practice, the heat method approximates solution to this particular problem from the heat kernel, namely the Green's function for the heat equation, in the limit, where 
dissipation time approaches zero (a relation between heat and distance known as Varadhan's formula [49]). For more details we refer to [50] and [51].

Consider now the 2-point BVP such that the solution we are looking for, satisfies the heat equation inside a region $\Omega=] \partial_{0} \Omega, \partial_{1} \Omega\left[\subset \mathbb{R}^{n}\right.$ and takes prescribed values at the boundaries:

$$
h\left(\partial_{0} \Omega, t\right)=a(t), \quad h\left(\partial_{1} \Omega, t\right)=b(t) .
$$

Then, according to the Eq. (11), we expect the temperature distribution $h$ to change with time. However, if $a(t)$ and $b(t)$ are both time-independent (i.e. if they are constant over time), then one might expect the solution to eventually reach a steady-state solution after a certain amount of time (i.e. limits for t approaching infinity) [52]. Therefore, $\frac{\partial h}{\partial t} \rightarrow 0$ and the heat equation can be safely reduced to the Laplace equation, $\Delta h=0$ inside $\Omega$.

In classical physics, Laplace's equation arises in the description of all kinds of conservative physical systems in equilibrium. In the field of study of Laplace's equation, namely potential theory, a potential is a scalar function whose gradient vector field is divergence- and curlfree. The gradient is then said to be a conservative vector field. Since the principle remains the same if we replace the electric potential with temperature, we propose to determine the temperature distribution under the condition of the thermal equilibrium. The solution $h$ is a smooth scalar function whose gradient describes in which direction and at what rate the temperature, or equivalently, the distance increases the most rapidly around a particular location. For instance, this solution has been directly employed as an approximation for the scalar distance function on a surface in [53]. Such an assumption can be well argued for approximating distance maps in-between two parallel plates. However, as soon as one of the two boundaries is slightly deformed, the restrictive unit length condition will be removed from the corresponding gradient vector field. To surmount this issue, and following the principle of the heat method, we will first normalize the gradient vector field of $h$ and then recover the true distance function from the resulting unit-length gradient vector field.

From another point of view, solving Laplace's equation subject to appropriate Dirichlet boundary conditions, is equivalent to solving the variational problem of finding a function $h$ that satisfies the boundary conditions and has minimal Dirichlet energy.

Definition Let $\Omega$ be an open subset of $\mathbb{R}^{n}$. And let $h: \Omega \rightarrow \mathbb{R}$ be a $C^{2}$ function over $\Omega$, the gradient's scalar Dirichlet energy of $h$ is defined by the following positive real number:

$$
\left.E[h]=\frac{1}{2} \int_{x \in \Omega} \| \nabla h(x)\right) \|^{2} d x
$$

where $\nabla h: \Omega \rightarrow \mathbb{R}^{n}$ denotes the gradient vector field of $h$. Further details, from a Riemannian point of view, are provided in the Appendix A.

\section{Well-posedness of the 2-point boundary value problem:}

Before defining the BVP to be solved in this work, we shall give a remark that a solution to Laplace's equation is uniquely determined if appropriate boundary conditions are posed. More generally, a BVP can be solvable if and only if the problem is well posed. In particular, 
the previously defined Dirichlet problem can be solvable, if and only if the boundaries are smooth curves/surfaces [54, 55].

To initialize the workflow, the binary mask of the shape is eroded with a cross-shaped structuring element which is best suited for fine structures. The choice of the structuring element is of great importance in preserving, as much as possible, the geometry and topology of any arbitrary shape. The objective here is to deal with sharp peaks particularly. In the following, we denote the eroded mask by $S_{e}$. Then, we perform the Principal Component Analysis (PCA) on $S$ (viewed as a point cloud where each voxel is considered as a point). The eigenvector corresponding to the largest eigenvalue gives the axis called the principal axis of Inertia. This axis intersects the shape surface at two points $p_{1}$ and $p_{2}$ (called the shape poles). To ensure that the bounding sphere will sufficiently enclose the shape and, thus, to prevent boundaries from overlapping, we use a surrounding sphere $S_{s}$ of radius $R=0.8 * l$ and whose center coincides with the shape centroid, where $l$ is the usual Euclidean length of the segment $\left[p_{1}, p_{2}\right]$. To determine the shape/sphere centroid, we use the median point which is a better midpoint measure for cases where a small number of outliers could drastically skew the average. At this level, all the shape surface points will be located between two non-intersecting boundaries: $\partial_{0} \Omega=\bar{S}_{s}$, and $\partial_{1} \Omega=S_{e}$, where $\bar{S}_{s}$ denotes the region outside the sphere.

In practice, we are looking for an elliptic twice-differentiable function $h: \mathbb{R}^{3} \rightarrow \mathbb{R}$ which satisfies the Laplace's PDE $\Delta h=\operatorname{div}(\operatorname{grad}(h))=0$ inside the region $\Omega=\overline{\partial_{0} \Omega \cup \partial_{1} \Omega}$, subject to the Dirichlet boundary conditions $h\left(\partial_{1} \Omega\right)=1$ and $h\left(\partial_{0} \Omega\right)=0$ (i.e. $h$ is the function that has minimal Dirichlet energy for all $x \in \Omega$, also called the harmonic interpolant in potential theory). The physical intuition behind is to determine the equilibrium heat distribution in a perfectly symmetric spherical room since the divergence of the gradient vector field corresponds to some kind of fluid flow. Implicitly, the shape surface $\mathcal{S}$ can be approximated in function of the solution $h$, by the isosurface:

$$
\mathcal{S}=\{x \in \Omega \quad \mid h(x)=c\},
$$

where $c$ is a constant in $] 0,1[$. Varying $c$ continuously from 0 to 1 will give the disjoint isosurfaces of the distance function. It is also important to keep in mind that the major problem in approximating geodesic distance is that the latter fails to be smooth at points in the cut locus, i.e. points that are equidistant from at least two points on a boundary. Fortunately, however, since the organ surface is smooth enough, then cut locus issues are avoided and the smoothness of the solution would not affect the geometry of the isosurfaces of the distance function.

\section{Numerical integration:}

To approximate the numerical solution of Laplace's equation, we use the Jacobi iterative

relaxation method which is simple to implement and which allows fast approximation of the 
solution in Cartesian coordinates:

$$
\begin{gathered}
h_{t+1}(i, j, k)=\frac{1}{2\left(\Delta i^{2} \Delta j^{2}+\Delta i^{2} \Delta k^{2}+\Delta j^{2} \Delta k^{2}\right)}\left(\Delta j^{2} \Delta k^{2}\left[h_{t}(i+\Delta i, j, k)+h_{t}(i-\Delta i, j, k)\right]\right. \\
\left.+\Delta i^{2} \Delta k^{2}\left[h_{t}(i, j+\Delta j, k)+h_{t}(i, j-\Delta j, k)\right]+\Delta i^{2} \Delta j^{2}\left[h_{t}(i, j, k+\Delta k)+h_{t}(i, j, k-\Delta k)\right]\right)
\end{gathered}
$$

where $t$ is the iteration index. In this work, we process reconstructed data with an isotropic voxel spacing of $1 \times 1 \times 1 \mathrm{~mm}$ (i.e. $\Delta i=\Delta j=\Delta k=1$ ).

\section{Convergence criterion:}

The scalar function $h$ is initialized to 0 inside $\Omega$ and then iteratively relaxed by finite differences until a satisfactory heat steady state solution is reached. A parallel computational implementation is performed in such a way that all grid points inside $\Omega$ are simultaneously visited at each iteration. A convergence criterion can be defined, through a discretization of the eq (14), by the following expression based on the total field energy over $\Omega$ :

$$
\epsilon_{t}=\sum_{\Omega} \sqrt{\left(\frac{\nabla h_{t}^{i}}{2}\right)^{2}+\left(\frac{\nabla h_{t}^{j}}{2}\right)^{2}+\left(\frac{\nabla h_{t}^{k}}{2}\right)^{2}}
$$

where $\nabla h_{t}^{i}=h_{t}(i+1, j, k)-h_{t}(i-1, j, k), \nabla h_{t}^{j}=h_{t}(i, j+1, k)-h_{t}(i, j-1, k)$,

and $\nabla h_{t}^{k}=h_{t}(i, j, k+1)-h_{t}(i, j, k-1)$. The Jacobi iterative computational scheme of eq (16) converges when the ratio $\epsilon=\frac{\epsilon_{t}-\epsilon_{t+1}}{\epsilon_{t}}$ becomes smaller than a user-defined threshold (typically about $10^{-5}$ ). Intuitively, this aims at encouraging smooth scalar fields by penalising large spatial gradients between neighbouring grid points. To speed up the algorithm, we strongly recommend to keep the number of iterations as a user defined parameter in order to avoid the repeated evaluation of $\epsilon$ at each iteration. A total number of 200 iterations is used for solving the Laplace's equation in this work.

\section{Computing the unit-length normal vectors to the tangent planes to the har- monic layers:}

As argued before, the gradient of $h$ is highly sensitive to errors in magnitude. We therefore compute the unit normal gradient field $N=-\frac{\nabla h}{\|\nabla h\|}=\left(N_{i}, N_{j}, N_{k}\right)^{T}$, so that the magnitude can safely be ignored when recovering distance maps from $N$ in the next step. The Eikonal equation will then automatically be satisfied thereafter, without being solved directly. The same operation was referred to as a change of variable in the heat method (the second step) [44]. Integrating this normal velocity vector field allows for a bijective mapping between pairs of points (i.e a one-to-one mapping). This gives a set of paths of minimum Dirichlet energy (i.e. a geodesic flow) for mapping the shape to a sphere. Let us underline that $N$ is also a conservative vector field (curl free) when evaluated at each isosurface $h=h_{\text {iso }}$. More details are provided in Appendix B. 


\section{Recovering geodesic distance maps:}

This last step resembles the third and last step in the heat method which consists of computing true distance functions (potential scalar function $L_{n}$ ) whose gradient is parallel to $N$. We therefore find the closest scalar potential $G=L_{0}+L_{1}$ by minimizing $\sum_{n=0}^{1} \int_{\Omega}\left|\nabla L_{n}-N\right|^{2}$, or equivalently, by solving the elliptic Poisson equation $\nabla \cdot \nabla L=\nabla \cdot N$, where $(\nabla \cdot)$ is the divergence operator, or even more simply, by aligning $\nabla L$ and $N$.

In the discrete setting, a solution to the above stated problem can be determined by solving a couple of Euler-Lagrange PDEs: $\nabla L_{0} \cdot N=-\nabla L_{1} \cdot N=1$, subject to the boundary conditions $L_{0}\left(\partial_{0} \Omega\right)=L_{1}\left(\partial_{1} \Omega\right)=0 . L_{0}$ and $L_{1}$ are first initialized to 0.5 and then iteratively updated inside $\Omega$ using a symmetric relaxation Gauss-Seidel method:

$$
\begin{aligned}
& \frac{L_{0}^{t+1}[i, j, k]}{\alpha}=1+\left|N_{i}\right| L_{0}^{t}[i \mp 1, j, k]+\left|N_{j}\right| L_{0}^{t}[i, j \mp 1, k]+\left|N_{k}\right| L_{0}^{t}[i, j, k \mp 1] \\
& \frac{L_{1}^{t+1}[i, j, k]}{\alpha}=1+\left|N_{i}\right| L_{1}^{t}[i \pm 1, j, k]+\left|N_{j}\right| L_{1}^{t}[i, j \pm 1, k]+\left|N_{k}\right| L_{1}^{t}[i, j, k \pm 1]
\end{aligned}
$$

where: $\left\{\begin{array}{l}m \pm 1=m+\operatorname{sgn}\left(N_{m}\right), \quad m \mp 1=m-\operatorname{sgn}\left(N_{m}\right) \\ \text { for } m \in\{i, j, k\}, \quad \text { and } \quad \alpha=\frac{1}{\left|N_{i}\right|+\left|N_{j}\right|+\left|N_{k}\right|}\end{array}\right.$

with: $\operatorname{sgn}($.$) is the sign function, L_{0}(x)$ is the length of the optimal geodesic path from the point $x=(i, j, k)$ to $\partial_{0} \Omega$, while $L_{1}(x)$ is the length of the optimal geodesic path from $x$ to $\partial_{1} \Omega$. The sum of these two lengths $G(x)=L_{0}(x)+L_{1}(x)$, defined as thickness in [45], represents in fact the length of the optimal geodesic path from $\partial_{0} \Omega$ to $\partial_{1} \Omega$ that passes through $x$. These geometric methods which are fully non-parametric allow one to handle time derivatives with finite differences in a restricted physical domain that exhibit large deformations. This step is also parallelized by simultaneously visiting all voxels inside $\Omega$ at each iteration.

\section{Proposed feature for surface characterization:}

Finally, we define a flexible feature by the following application:

$$
\begin{gathered}
\tilde{f}: \Omega \rightarrow \mathbb{R}_{+}^{*} \\
x \mapsto \frac{R}{G(x)}
\end{gathered}
$$

Note that $L_{0}>>L_{1}$ for all the surface points so that $G \simeq L_{0}$ and only one PDE has to be solved to calculate the feature function (see Fig. 3.c, 3.e). The function $\tilde{f}$ has the potential to characterize the surface variation and to delineate between concave, convex and flat regions 
as illustrated in Fig 4 for both torus and ellipsoid. Relatively, the largest feature values correspond to the most convex areas in the surface while the smallest values correspond to the most concave areas. The proposed feature values exhibit smooth transitions between concave and convex regions. Furthermore, this feature is scale-invariant thanks to the use of variable sphere radius, rotation-invariant thanks to spherical symmetry, and also invariant to translation since the sphere and shape are concentric. An illustration of all the previous steps is presented in Fig. 3. Fig 4 illustrates the obtained feature maps for a set of symmetric and non-symmetric geometries.
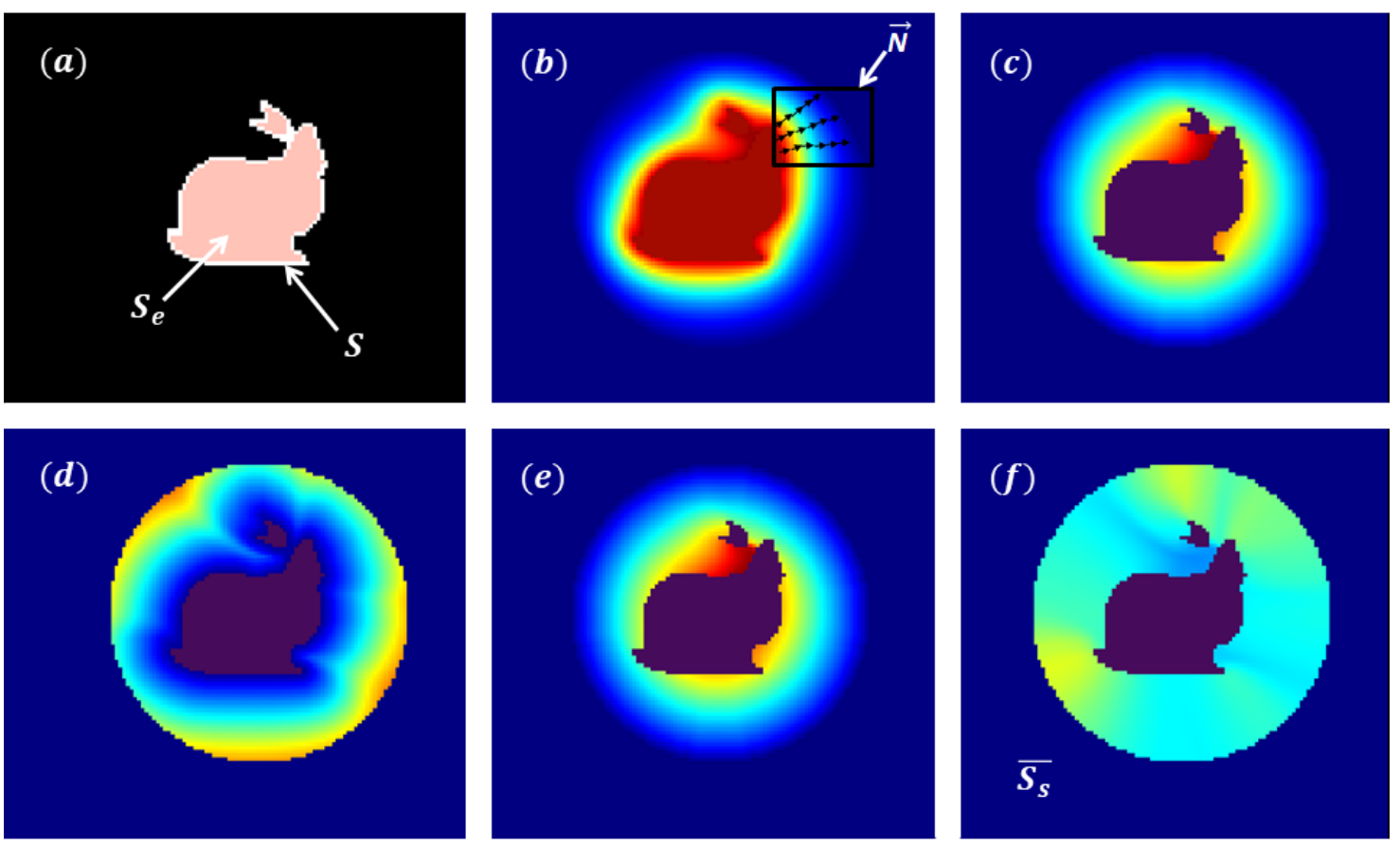

Figure 3: Proposed method (Stanford Bunny example): (a) binary mask and its eroded version, (b) harmonic map: heat values range from blue (0) to red $\left(10^{4}\right)$, (c) length of the geodesic path from $x$ to the surrounding sphere $L_{0}$, (d) length of the geodesic path from $x$ to the inner boundary $L_{1}$, (e) length of the resulting geodesic path $G$ (lengths are expressed in $\mathrm{mm}$ ), and (f) feature values $\tilde{f}$ between the two boundaries (in $\left.\mathrm{mm}^{-1}\right)$.

The ability to quantify organ shape changes with respect to the sphere would not only allow for deeper understanding of organ motion but also conceivably allow for improved detection of pathologies. In practice, many pathologies are associated with localized organ malformations. Fig. 5 provides evidence that the proposed feature is capable of discriminating such phenomena. Moreover, assuming that the organ volume should be preserved during motion, we suppose that the sphere radius should remain constant which is a sufficient condition to detect all local changes without any small scale effect. Therefore, the feature values, being inversely proportional to a distance measure, can be expressed in $\mathrm{mm}^{-1}$. 

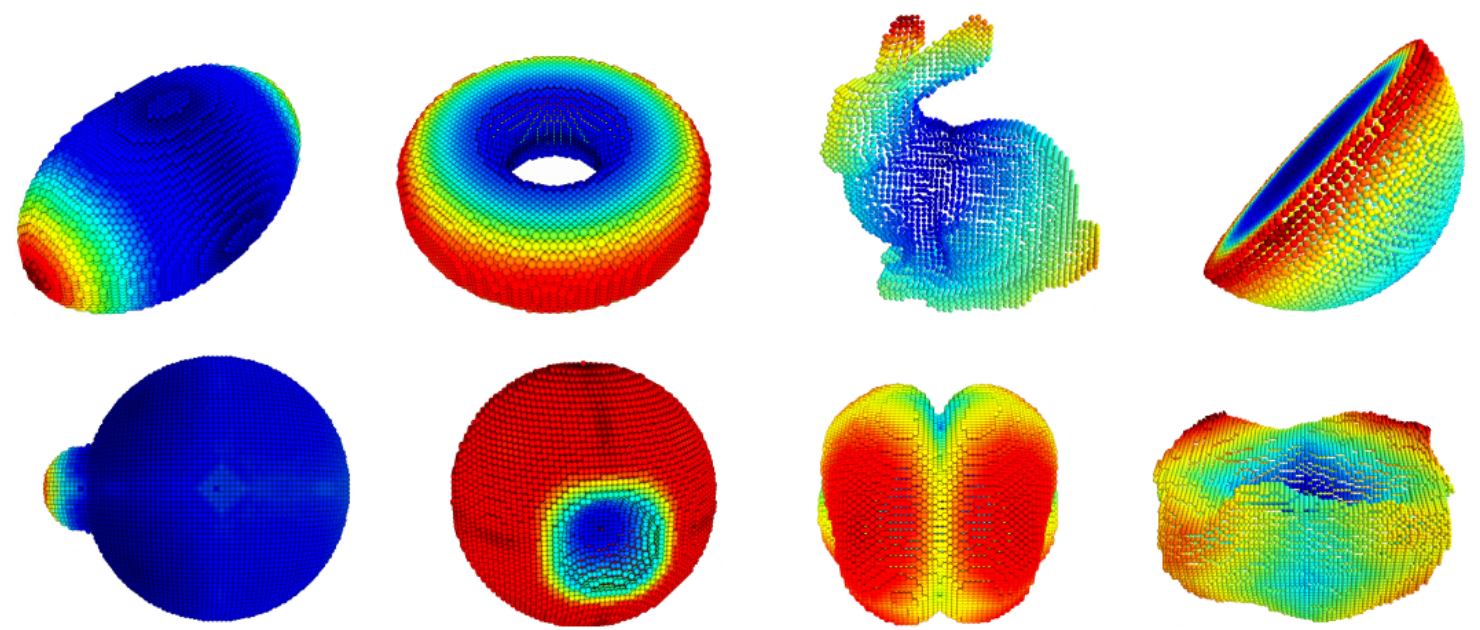

Figure 4: Obtained feature maps (normalized) for synthetic and realistic (e.g. neonatal brain and bladder) surfaces. Colormap goes from blue (0) to red (1).
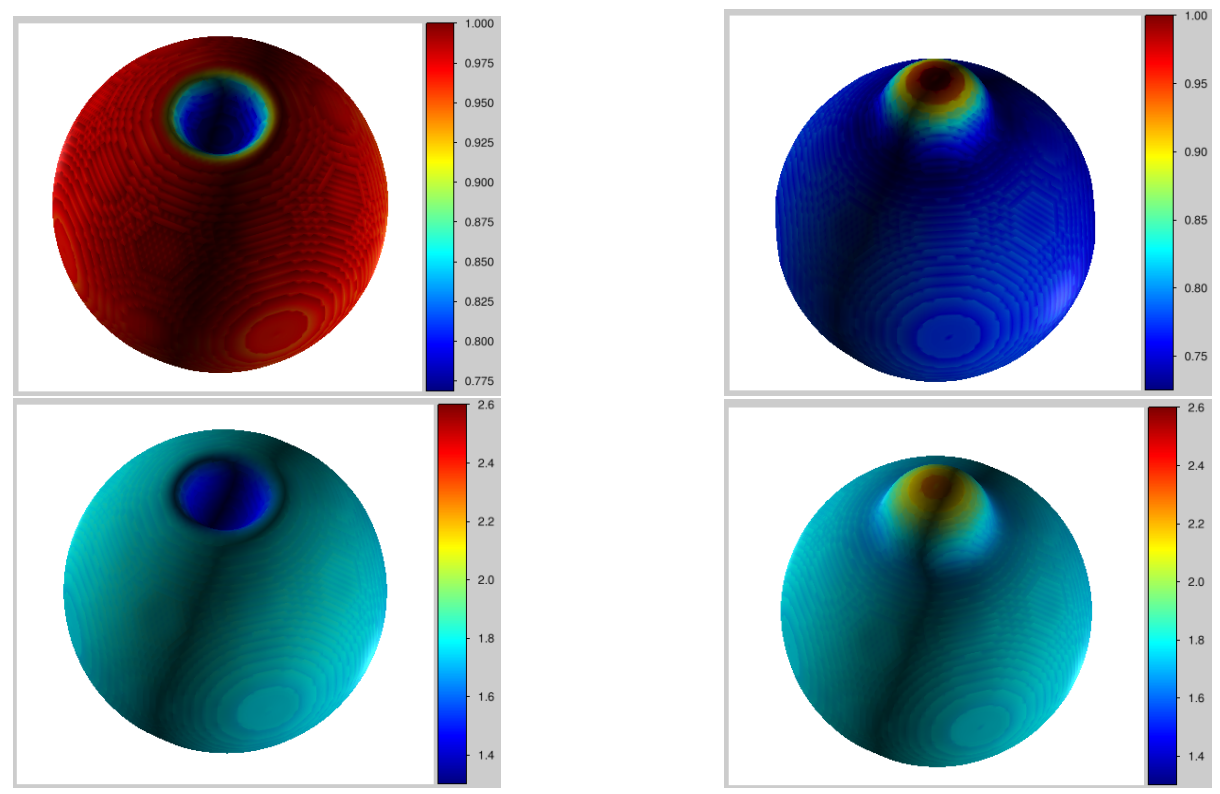

Figure 5: Capacity to detect local changes in surface geometry: first row (after normalization), second row (without normalization and with a commun truncated colorbar). Feature maps are plotted on the extracted isosurface $h=0.98$.

\section{Experiments and Results}

\subsection{Data set}

\subsubsection{Realistic dynamic MRI data}

Pelvis areas of seven healthy participants (five women), ranging in age from 23 to 31 years, and in weight from 58 to 81, were imaged. Subjects were imaged with a $1.5 \mathrm{~T}$ MRI 
scanner (MAGNETOM Avanto, Siemens AG, Healthcare Sector, Erlangen, Germany) using a spine/phased array coil combination and $T_{1} / T_{2}$ weighted balanced steady-state free precession sequences $\left(T_{1} / T_{2} W\right.$ bSSFP). A quasi-isotropic 3D static image was recorded during a maximum expiration apnea of 18 seconds. Multi-planar dynamic acquisition assuring full coverage of the pelvic region was recorded during a $80 \mathrm{~s}$ forced breathing exercise. During this exercise, the subject alternately inspired and expired at maximum capacity. Subjects were also instructed to increase pelvic pressure at the maximum inspiration and then to contract abdominal muscles during the expiration. These actions increased the intra-abdominal pressure, causing deformations of the pelvic organs. Spatial configuration of the multi-planar acquisition is described in detail in [9]. The study was approved by the local human research committee and was conducted in conformity with the Declaration of Helsinki. Since no extraneous liquids was injected into pelvic cavities in this study, only the segmentation of the bladder was performed and the analysis focused exclusively on this organ. For each subject, the three-dimensional dynamic sequences acquired in multi-planar configurations allowed the reconstruction of nearly 400 bladder volumes generated at a rate of 8 volumes per second. Bladder volume of each subject was computed from the manual segmentation of the static acquisition. Bladder volume presented a large variability among subjects (values were ranged from $48 \mathrm{~cm}^{3}$ to $403 \mathrm{~cm}^{3}$ ). Since the scanning duration is short relative to the organ motion, spatio-temporal reconstructions were performed outside the MR scanner to recover the missing data using diffeomorphic registration as detailed in [9].

\subsubsection{Data simulation}

To evaluate the capability of each geometric descriptor in detecting abnormalities in bladder dynamics during breathing exercises, we have simulated a smooth continuous-time trajectory of the organ volume using a Log Euclidean Polyaffine registration framework [36] to excite the organ deformation from the interior with locally controlled properties. The organ volume in the static scan is divided into four non-intersecting regions and an affine transformation is associated to each region or component (we have only used component-wise scaling transforms in such a way that the organ volume is still preserved throughout the sequence). A first motion cycle is simulated by estimating a flow of invertible diffeomorphisms for mapping the organ volume from resting state (i.e. organ volume in the stationary scan) towards the maximum of inspiration state exhibiting the largest deformation. A forward trajectory was estimated based on the integration of stationary velocity fields via the exponential map. Then, the inverse trajectory for coming back to the resting state was obtained by smoothly interpolating the inverse of the simulated polyaffine transformations. Finally, this motion cycle is repeated 8 times for stability assessment of different measures.

In practice, we simulated a large deformation to make the organ fall down by maximum of inspiration which allow the floor of the bladder to sag through the muscle and ligament layers. This abnormal kind of motion occurs frequently in women with uterine and bladder prolapse for which the bladder can create a bulge into the vagina because of the weakness of their pelvic muscles and ligaments. Fig. 6 illustrates the simulated organ trajectory which we will call Simulated Pathology (SP) in the sequel. This sequence, for which the shape trajectory is well known, will serve to compare the different features used in this study in 
terms of stability and measure repeatability during forward organ movement. It will also serve to assess their capacity to detect this abnormal motion type in a mixed data set.

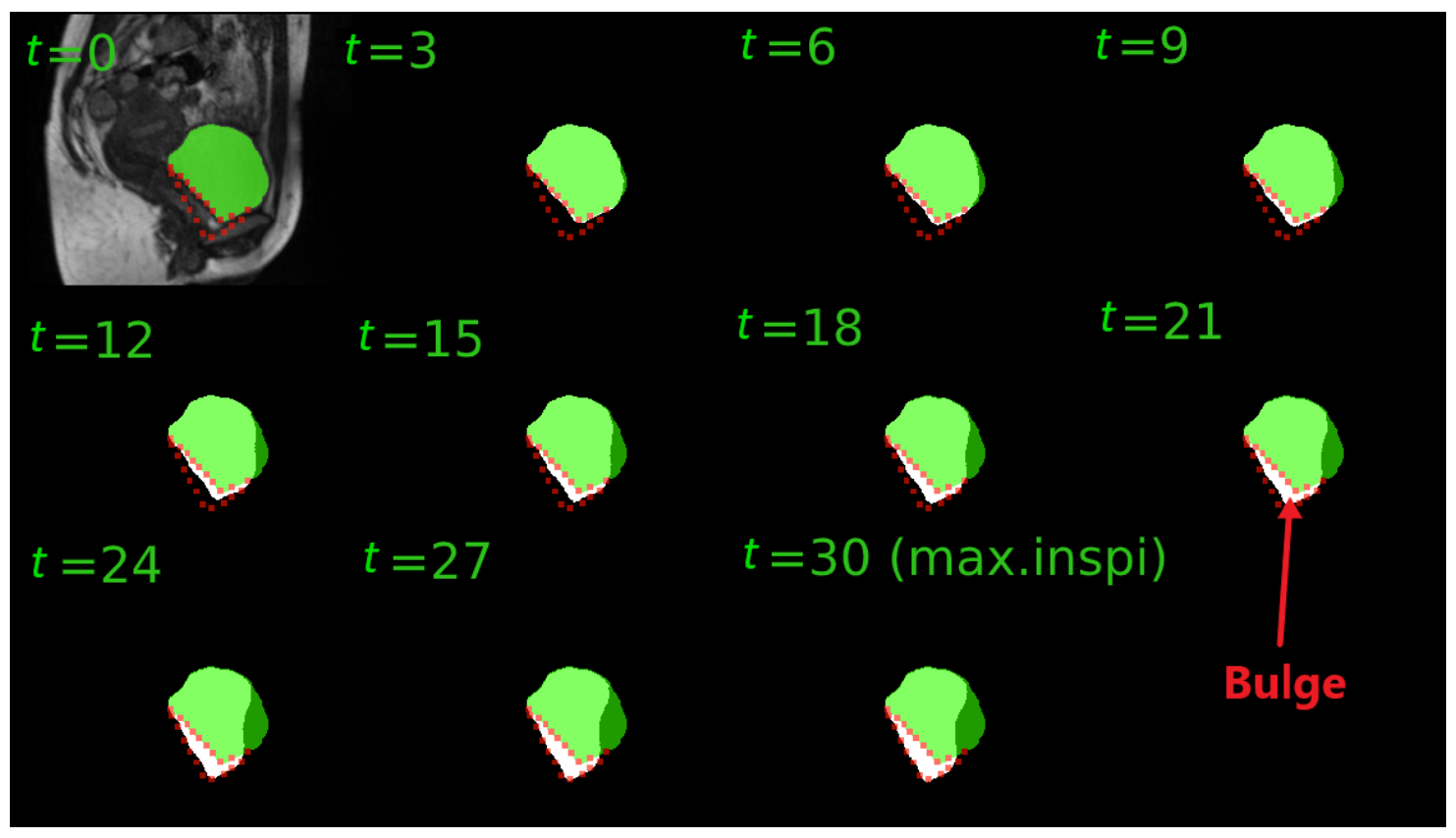

Figure 6: Simulation of large nonlinear deformations using the LEPF: the green mask corresponds to the bladder segmentation at the resting state, the white segmentations correspond to the temporal organ positions, while the red dotted area shows the regional bladder motion abnormality (the resulting bulge through the vaginal canal).

\subsection{Tracking of mesh vertices}

Diffeomorphic registrations were performed using the software package Deformetrica [56]. The parameters of the registration algorithm were set as follows: the standard deviation of the Gaussian kernel defined in (1) was set to $\sigma=10^{-4}$ in order to obtain a deformation field with a narrow precision. The kernel-width parameter for controlling the granularity of the deformation was set to 8. 15 intermediate states describing the temporal evolution of the tracked points were estimated in order to obtain a smooth continuous-time trajectory of the organ between successive time frames. The loss function defined in (5) was minimized using the gradient descent optimization method. All experiments were performed on an Intel ${ }^{\circledR}$ Xeon ${ }^{\circledR}$ Processor Silver 4214 CPU @ $2.20 \mathrm{GHz}$, with a physical memory of 96GB. For the first subject for example, Algorithm 1 took 6 sec to align a set of 342 tracked point set $\mathcal{M}_{t}$, with the target set $\mathcal{C}_{t}$, composed of 5686 points for which only a set of 210 control points have been used for optimizing the shape matching.

\subsection{Geometric descriptors}

In this study, we have used different shape descriptors that can be classified into two categories: 
- Biomechanical descriptors: the deformations were quantified using mesh temporal elongations and distortions. This aimed to extend the methodologies used in [37] from $2 \mathrm{D}+t$ to $3 \mathrm{D}+t$.

- Geometric descriptors (family of geodesic-based features): the mean curvature and the new proposed descriptor which take into account the non Euclidean geometry of three dimensional shape space.

A comparison between descriptors was performed where the goal was to assess their robustness by performing trajectory stability and sensitivity analysis.

In fact, the propagated tracking-errors may affect the mesh regularity in the neighbourhood of some slightly perturbed vertices. Such small errors can be from any source. For instance, they may have originated from velocity smoothing within the LDDMM, which can also be mixed with round-off errors propagated in the numerical integration of differential equations when computing the features themselves. Assuming that the effect of error propagation increases with time, neighbourhood-based features may thus be more or less sensitive to these cumulative errors. To evaluate the robustness of each descriptor with respect to error propagation, we computed the following error ratio that is indicated in Fig. 7:

$$
E=1-\operatorname{normcorr}\left(\mathcal{F}_{r}(0), \mathcal{F}_{r}\left(t_{\max }\right)\right)
$$

where normcorr $(.,$.$) is the normalized correlation function, \mathcal{F}_{r}(0)$ is the reference feature map, and $\mathcal{F}_{r}\left(t_{\max }\right)$ is the obtained feature map at the last time the resting state is revisited. Error estimates for the characterization of simulated organ shape trajectory were: 0.045 for mesh elongations and distortions, 0.034 for Riemannian mean curvature, and $3.10^{-6}$ for the proposed feature.

Fig. 7 shows different feature map dynamics across time for the simulated sequence. The top meshes represent the shape at each revisited resting state. Using the geodesicbased feature for which the computations were totally independent of vertex neighbourhood characteristics, the feature map differences were very close to zero over all the surface but we can observe the presence of small feature changes arising around some vertices, caused mainly by propagation of neglectable registration errors. This means that this feature is capable of detecting these perturbations as well as providing correct correspondence trajectories in a bijective fashion. Moreover, the use of the proposed feature exhibited a perfect correlation curve repeatedly showing a correlation value which is very close to 1 at each revisited resting state.

To evaluate descriptors in terms of their capacity to detect motion abnormality, we have defined the deformation depth parameter by Depth $=1-$ mincorr, where mincorr is the minimal correlation value achieved at maximum of inspiration (see Fig. 7). The obtained deformation depths were: 0.12 for mesh elongations, 0.115 for mesh distortions, 0.17 for Riemann mean curvature, and 0.36 for the proposed feature. Since we have simulated a large deformation in a specific direction (see Section 4.1.2), it would be expected that the corresponding correlation trajectory will exhibit an important deformation depth. Which was the case using our descriptor, in a more remarkable way. 
On the other hand, sensitivity assessment consisted of testing the robustness of featurebased statistical characterizations in the presence of some uncertainties related to the data acquisitions. For example, respiratory depth and rythm may vary slightly over time and cannot be totally controlled during MR scanning. Therefore, we cannot assure that the resting state will reappear somewhere else in the sequence while preserving exactly the same initial organ shape patterns. To compare between descriptors in this context, we used a realistic organ trajectory, for which explicit error models are not known.

Fig. 8 shows that the correlation curve associated to the proposed feature is characterized by greater spatio-temporal stability than those produced by employing each of the other descriptors for characterizing one realistic organ shape trajectory. These experiments show also that mesh elongations are very sensitive to noise and tracking-error propagation which makes it difficult to quantify breathing frequency. It is also clear that the stability of distortion measures decreases significantly with time. This confirms the assumption mentioned above and also confirms the fact that the geodesic-based features are more capable of describing respiratory frequency and depth.

\subsection{Subject-specific organ dynamics}

Results presented in Fig 9 illustrate the organ motion patterns for all subjects using the proposed descriptor. For each motion cycle, a decrease in correlation coefficients reflects changes in surface geometry, relative to the reference state. This can be interpreted by the fact that forced inspiration involves an action of the diaphragm and abdominal muscles which induces deformation of the internal organs. Otherwise, when the correlation values increase, the patient releases the pressure and consequently the bladder relaxes and returns to its initial shape. Respiratory motions were expected to be perfectly regular during scanning. However, depth and rhythm may vary over time. The patient's breathing patterns then becomes irregular with time which can be noticed from the correlation trajectories. For all healthy subjects, the organ was highly deformed by maximum of inspiration and the deformations occured essentially in the top lateral regions. In terms of motion patterns, the organ shape at the maximum of expiration state was very close to the shape at resting state, especially for subjects $S_{1}, S_{4}$, and $S_{5}$ (see top meshes in each subfigure). Clearly, the resting state has never been revisited throughout the organ shape trajectory for the other subjects. This can explain the reduction in correlation across cycles.

\subsection{Inter-subject variability in motion patterns}

In addition to the organ shape inter-variability, depth and rhythm of respiration may vary across subjects. Depending on individual lung functions and capacities, healthy individuals exhibit different motion patterns and thus different bladder volume changes. In this work, inter-subject comparisons were performed in two ways: i) a global metric is achieved using the organ deformation depth which reflects the respiratory depth (section 4.5.1). And ii) a

more fine comparison is performed by establishing point-to-point anatomical correspondences to compute inter-correlations by means of topological characteristics (section 4.5.2). 


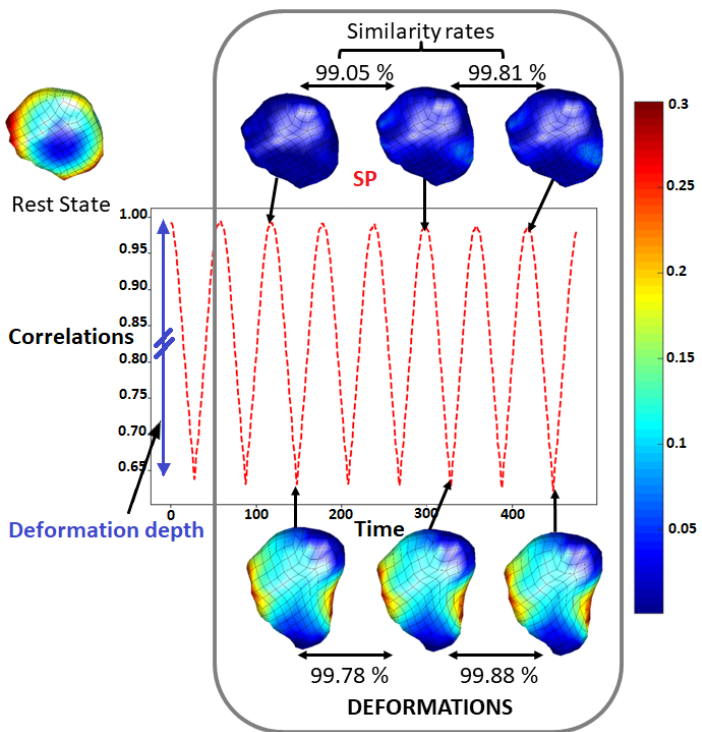

(a) Proposed feature (in $\mathrm{mm}^{-1}$ )

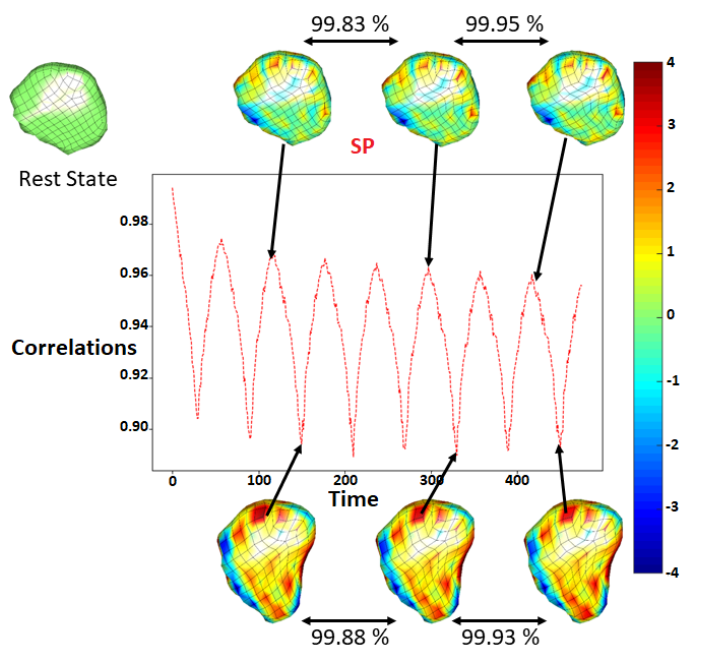

(c) Mesh elongations (in $\mathrm{mm}$ )

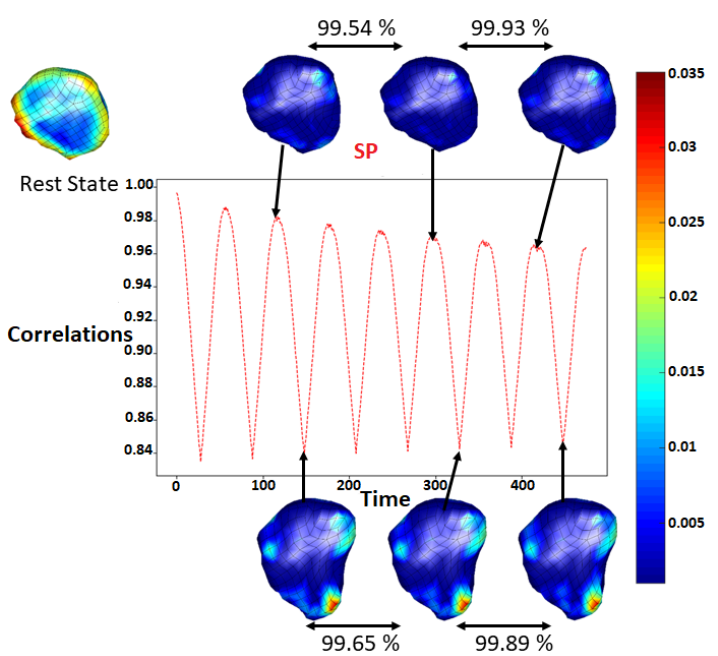

(b) Mean curvature (in $\mathrm{mm}^{-1}$ )

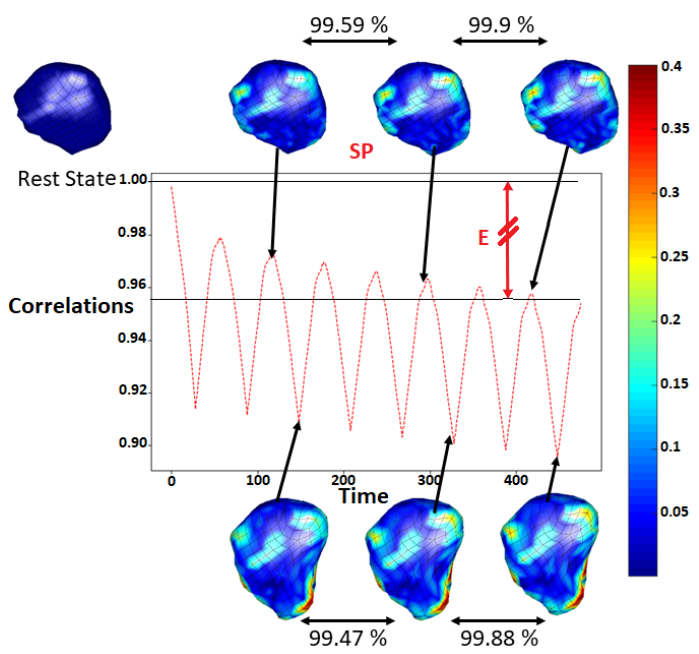

(d) Mesh distortions (in degrees)

Figure 7: Feature map dynamics (changes) w.r.t the resting state for the synthetic sequence. Elongations and distortions showed the largest sensitivity rate to tracking-error propagation: $E=4.5 \%$. Results show up to $99 \%$ correlation (similarity rate) between feature maps at first and last (9th) resting states for our descriptor, more than $3 \%$ better than those of other descriptors. For (a), (b) and (d), colorbars range from blue (no deformation) to red (high deformation). For (c), the green color indicates that no deformation has occured.

\subsubsection{Maximal-deformation based comparison}

Fig 10 depicts the normalized distance matrices based on the absolute differences of deformation depths between each pair of subjects. We have introduced the simulated sequence into the data set for comparing between two different classes of motion, i.e. normal and 


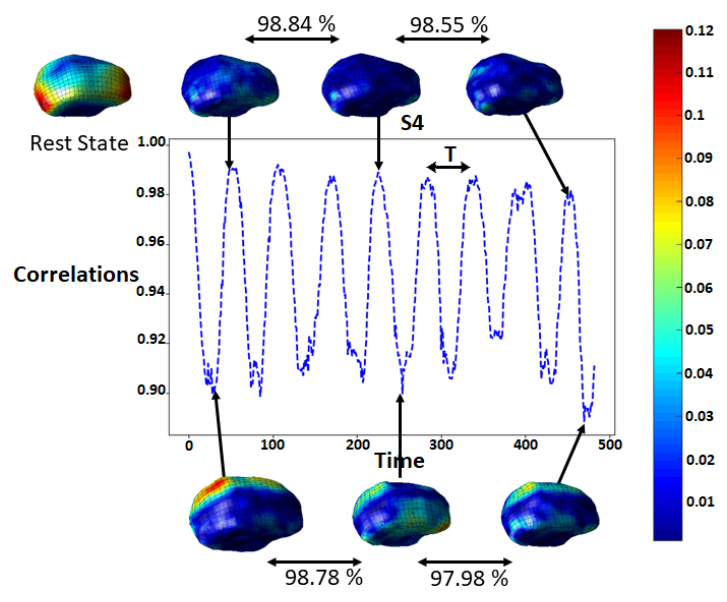

(a) Proposed feature (in $\mathrm{mm}^{-1}$ )

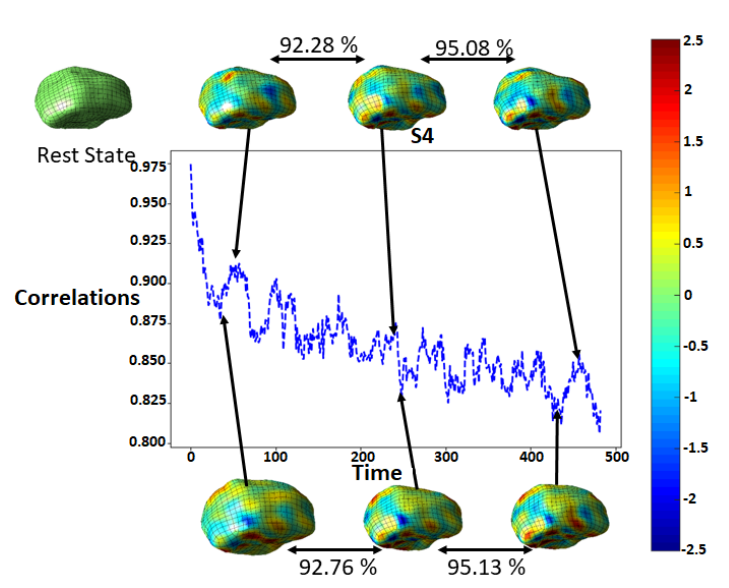

(c) Mesh elongations (in $\mathrm{mm}$ )

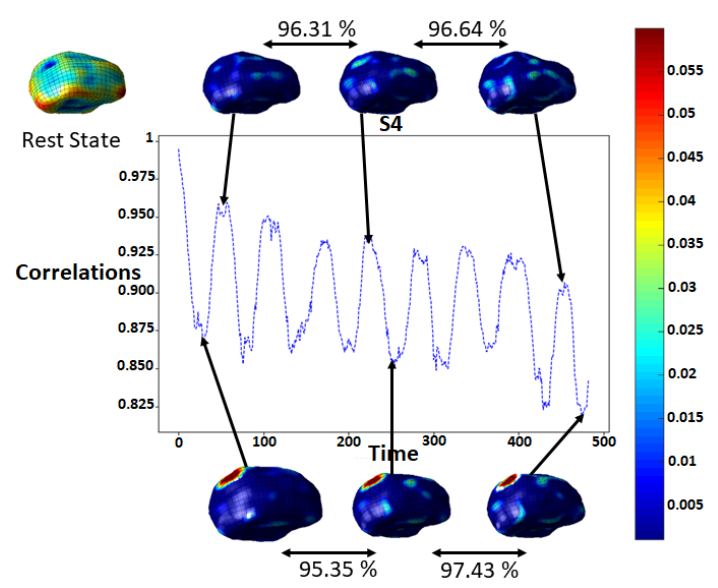

(b) Mean curvature (in $\mathrm{mm}^{-1}$ )

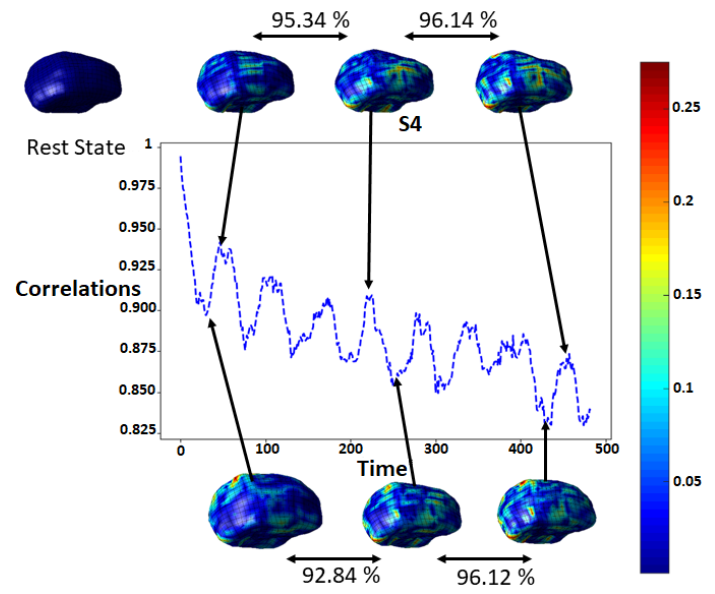

(d) Mesh distortions (in degrees)

Figure 8: Feature map dynamics (changes) w.r.t the resting state for one realistic organ shape trajectory. As shown in (a) and (b), a fine characterization of organ shape trajectory using geodesic-based features can allow for approximating the respiratory frequency, $f_{r} \approx \frac{1}{T}$, where $T$ is the median time interval between two successive maximums of the correlation curve. For (a), (b), and (d), colorbars range from blue (no deformation) to red (high deformation). For (c), the green color indicates that no deformation has occured.

abnormal. The objective of these experiments is then two-fold: 1) to show how deformation depths vary across healthy subjects, depending on their individual breathing capacities (left panel), 2) to evaluate the capacity of each descriptor to filter out the introduced artificial data from the mix, and thus to recognize any kind of abnormal motion, such as the pathological motion type described in Section 4.1.2 (right panel). As illustrated in the figure, the artificial sequence was poorly correlated with the realistic ones in terms of deformation depth. This difference was significant when using the proposed feature and the Riemannian curvature which makes them better suited as classifiers, but less significant when using mesh distortions and elongations for the characterization of organ dynamics. These results are in 
line with those obtained in previous sections 4.3 and 4.4 .

\subsubsection{Inter-subject comparison using the spherical parameterization}

Because of the large inter-variability between subjects in terms of organ volume and geometry, the organ surface parameterization differed across subjects (see Section 3.1). We then used the eigenfunctions of the LBO to find point correspondence between two vertex sets in order to compare between different feature-based characterizations. Each subject $X$, for which the organ surface was encoded with $n$ samples, was compared with each other subject $Y$ in the database with a unique parameterization (that of $X$ ). We therefore adopted the spherical parameterization method introduced in [57]. This method considers only the three first non-trivial eigenfunctions of the LBO of the closed (genus-0) surfaces. However, it is not guaranteed that the three first eigenfunctions possess only two nodal domains which may lead to some irregular situations such as singular vertices in sphere-mesh. To deal with this problem, we followed [58] by taking into account the six first eigenfunctions and then selecting among them the first three eigenfunctions with only two nodal domains. Further details of the methods are provided in the Appendix C.

For the proposed geodesic-based feature, the spherical mapping was straightforward since we have the geometric flow that maps the mesh vertices to their corresponding vertices on a sphere surface (see Appendices A and C for more details), and only the surface resampling (i.e. the next step) was required to compute inter-correlations between individuals' motion patterns.

The second step consisted of measuring similarities between individual's motion patterns (i.e. between the individual time-average feature vectors) using a temporal inter-subject comparison (vertex-to-vertex) based on a spherical interpolation of vertices and their corresponding texture values in the unit sphere using the Kdtree interpolation approach for surface resampling, included in the Spherical Demons framework [59].

Fig. 11 shows the quality of feature projections on the unit sphere for the simulated sequence. In clinical practice, such projections will provide full $4 \mathrm{D}$ information using a common and simple shape representation to easily localize the most important and common deformations at the population level.

Fig. 12 illustrates the complete pipeline for measuring the similarity between two surfaces with different resolutions based on the normalized proposed feature. All the results of intersubject comparison using LBO spherical parameterization are given in Fig. 13. These results are consistent with those obtained using deformation depth as a global metric for intersubject comparisons. They confirm the ability of our descriptor to discriminate between normal and abnormal motion.

\section{Discussion and conclusion}

\subsection{Comparison with related works and their clinical relevance}

Actually, only 4D simulations (biomechanical models) of the 4D pelvic organ motion are available in the literature $[7,8]$. This work represents a research initiative that aims to assess the 4D motion of such organs from realistic observations using noninvasive imaging 
techniques. Hence, the data reconstruction itself reveals clinically relevant insights into the understanding of pelvic floor dynamics. Furthermore, there is a need for a consensus on the image acquisition protocols themselves [4]. And the results of the current work will be used to adjust the current acquisition protocols for dynamic MRI sequences to make them more exploitable and well adapted for 3D reconstruction and post-processing. This study represents an improved extension of the works of [37] from $2 \mathrm{D}+t$ to $3 \mathrm{D}+t$ for characterizing pelvic floor organ dynamics. In $2 \mathrm{D}+t$ studies, the out of plane problem related to the acquisition protocols and conditions can lead to incomplete or even misleading interpretations, which can prohibit their use in clinical settings. Moreover, this natural extension was required because of the variability of abdominal organ shapes across time and subjects. To address these competing concerns, statistical shape analyses were performed in the high resolution domain to allow quantification of the $4 \mathrm{D}$ motion of the bladder. Given the fact that this extension has pointed out that the bladder deformations occur essentially in top lateral regions, then it becomes obvious that this valuable information cannot be directly extracted from dynamical sagittal slices. This makes it unnecessary to perform a direct comparison between the two studies.

In terms of methodologies and tools, the novelty in this work is that we characterize the organ dynamics only from surfacic information (i.e. using surface meshes) contrary to finite element modeling for stress analysis using volumetric meshes which also discretize the interior structure of the organ $[7,8]$. Our fine characterization can be clinically useful to measure respirations indirectly through the deformation induced by their actions on bladder shape: in addition to the assessment of respiration depth that can be deduced from the deformation depth parameter, Fig. 8 illustrates its use as a promising tool for identifying subject-specific respiratory frequencies by exploiting properties of periodicity in correlation trajectories.

\subsection{Surface representation and motion characterization}

In this study, we combine parametric methods (LDDMM, for surface representation) and non-parametric methods (an Eulerian PDE approach, for surface characterization). The LDDMM was employed to represent the organ surfaces as 4D smooth quadrilateral meshes, empty from irregular situations such as singular vertices near the organ shape poles, as encountered in [7]. The idea behind was to parameterize large deformations with only representative samples of surface points encoding the major shape variability with reasonable computational costs within large datasets. The use of the LDDMM, employing Hamiltonian statistical mechanics, aimed at providing an hypothesis compatible with the physics of deformations. This helped for establishing a compact shape representation relying on structured meshes for the organ surface with fixed connectivity information. A set of 3D geometric descriptors was employed to extract meaningful features characterizing the bladder shape dynamics during loading exercices. Elongations and distortions are a well known biomechanical parameters which have been used in [37] to classify subjects into pathologi-

cal patients and healthy control groups. However, since we would like to characterize the geometry of highly curved organs, then statistical tools derived from Euclidean geometry are not the most appropriate to deal with such clinical issues. In addition to the extension 
of elongations and distortions from 2D to 3D, we have also employed two shape descriptors involving the notion of geodesic distances for a finer characterization of organ dynamics: the mean curvature and our proposed feature were used to identify salient motion patterns.

To study the temporal changes in mean curvature, we have used the method proposed in [60], to estimate curvatures on triangle meshes. This required the conversion of temporal quad meshes to triangular ones and the major limitation was the dependency of the algorithm on mesh resolution and vertex neighborhood size (this algorithm is well adapted to highresolution meshes). To cope with this dependency, we proposed the use of a fully nonparametric Eulerian PDE approach to explore the space of continuous maps from shape surface into a surrounding sphere surface.

To analyze descriptors' performances, we have deformed the bladder volume in the static MRI scan for one subject to simulate a biologically plausible model of abnormal shape trajectory using the LEPF (this aimed to locally control the deformation kinematics, while providing globally nonlinear deformation). The results of our experiments on several descriptors show the effectiveness of the proposed shape descriptor to characterize surface dynamics through its application to this cyclic trajectory, characterized by its large deformation-depth and long-term time variations (see Fig 7).

Both these results on synthetic data and the results illustrated in Fig 8 for characterization of a realistic organ shape trajectory show that the numerical stability of elongation and distortion measures decreases drastically across cycles. These examples also show that the proposed feature is numerically much more stable than tensor-based mean curvature which confirms the findings of Nava-Yazdani et al. [32] that computing tensors impacts numerical stability in time-dependent shape data analysis. In fact, mean curvature is an extrinsic measure of curvature which corresponds to layman's understanding of curvature before we were ever introduced to differential geometry. It is often employed in the Level set method for curvature-driven segmentation tasks [61]. Its expression for non-parametric surfaces is also known as the divergence formula [62]. In [63], Albin et al. showed that curvature estimates from implicit surfaces (for which the computations are performed in Cartesian coordinates) are more accurate than those calculated from meshes. This can explain the fact that nonparametric methods are more accurate and stable than parametric ones. In the same context, we have studied the sensitivity of parametric methods to mesh quality for estimating brain curvature tensor in [64]. In appendix A, we show that the non-parametric approaches can successfully avoid the need to compute metric tensors explicitly while taking into account the non-Euclidean geometry of organ curved surfaces.

On the other hand, comparisons between two or more patients may help identify movement abnormalities and may serve for subject classification: into healthy and pathological, or into subgroups, sharing similar organ motion characteristics within large data sets.

An inter-individual comparison is then performed by introducing the simulated sequence to the realistic dataset. A global metric is first achieved using the deformation depth. It reflects the maximum breathing capacity of each subject since the organ surface deforms the most by maximum of inspiration. As illustrated in Fig. 10, a quantification of subject-specific defor- 
mation depths during deep breathing exercices is capable of determining differences between patients (left panel) and groups (right panel). This may serve to optimize both surgical and non-surgical treatments of pelvic floor disorders with respect to individual breathing capacities. To make this comparison more statistically meaningful, local motion patterns derived from different descriptors and for different subjects (different parameterizations) were compared in a common space, the unit sphere, through the use of the LBO eigenfuctions for spherical mapping. Results in Figures 10 and 13 illustrate the fact that motion patterns differ across healthy volunteers and highlight the capacity of each descriptor to distinguish the simulated abnormal motion type from statistical measures. The results show also that the geodesic-based features (including curvature), for which the simulated sequence was less correlated with realistic ones, are the best classifiers against traditional classifiers based on Euclidean geometry. In fact, each feature have described an organ shape trajectory by integrating over time all the available information from each frame. However, we observed that for a deformation characterization task, not all frames contain salient spatio-temporal informations which are discriminative to different classes of deformations. Indeed, many frames contain non-salient motions which are irrelevant to the performed deformation. The characterization of the simulated trajectory represents a good example to illustrate these findings (Fig. 7). See also Fig. 8, in which mesh distortions and elongations provided more "non-salient" motion patterns, thus affecting the quality of the corresponding correlation trajectories.

\subsection{Limitations of the study and future scopes}

Although the fact that we propose a pipeline with minimal user intervention, let us recall that it is not yet fully automatic, since it requires manual segmentation of the acquired dynamical slices used for the reconstruction of organ volumes. Furthermore, it is required to establish only the initial quad mesh for the organ surface using Instant meshes. Concerning the proposed descriptor, we are considering only isotropic volumes for now.

Future work will be towards addressing these limitations with the help of deep learning approaches for slice segmentation [65], and to fully integrate the open source software of Instant Meshes [39] in a way to make the entire pipeline easy to handle for physicians and nurses. All the source codes developed and used in this work are available at https: //github.com/k16makki/dynPelvis/tree/master/Dynpelvis3D.

Future works will also include clinico-pathological data from age-matched women with uterine and bladder prolapse. The proposed methods will be employed to identify morphological differences between normal and pathological groups. These techniques could also be applied to study and characterize the dynamics of other functional human tissues and organs such as the heart. While it cannot be inferred from this study, it seems reasonable to hypothesize that the proposed tools can be employed to perform longitudinal analysis during organ disease development or following a therapy for pelvic floor disorders.

\section{Declaration of Competing Interests}

The authors declare that they have no conflict of interest. 


\section{Acknowledgments}

This research was supported in part by the AMIDEX - Institut Carnot STAR under the DynPelvis3D grant.

\section{References}

[1] Y. M. Law, J. R. Fielding, MRI of pelvic floor dysfunction, American Journal of Roentgenology 191 (2008) S45-S53.

[2] R. F. El Sayed, C. D. Alt, F. Maccioni, M. Meissnitzer, G. Masselli, L. Manganaro, V. Vinci, D. Weishaupt, ESUR, E. P. F. W. Group, et al., Magnetic resonance imaging of pelvic floor dysfunction-joint recommendations of the ESUR and ESGAR Pelvic Floor Working Group, European radiology 27 (2017) 2067-2085.

[3] A. Jourdan, A. Le Troter, P. Daude, S. Rapacchi, C. Masson, T. Bège, D. Bendahan, Semiautomatic quantification of abdominal wall muscles deformations based on dynamic MRI image registration, NMR in Biomedicine (2021) e4470.

[4] M. Garetier, B. Borotikar, K. Makki, S. Brochard, F. Rousseau, D. Ben Salem, Dynamic MRI for articulating joint evaluation on $1.5 \mathrm{~T}$ and $3.0 \mathrm{~T}$ scanners: setup, protocols, and real-time sequences, Insights into Imaging 11 (2020) 1-10.

[5] P. Chen, R. J. van Sloun, S. Turco, H. Wijkstra, D. Filomena, L. Agati, P. Houthuizen, M. Mischi, Blood flow patterns estimation in the left ventricle with low-rate 2D and 3D dynamic contrast-enhanced ultrasound, Computer Methods and Programs in Biomedicine 198 (2021) 105810.

[6] Z. Gorji, A. A. Pourmomeny, The effect of pelvic floor muscles training using biofeedback on symptoms of pelvic prolapse and quality of life in affected females, International Journal of Biomedicine and Public Health 3 (2020) 5-9.

[7] Z.-W. Chen, P. Joli, Z.-Q. Feng, M. Rahim, N. Pirró, M.-E. Bellemare, Female patientspecific finite element modeling of pelvic organ prolapse (POP), Journal of biomechanics 48 (2015) 238-245.

[8] H. Courtecuisse, Z. Jiang, O. Mayeur, J. Witz, P. Lecomte-Grosbras, M. Cosson, M. Brieu, S. Cotin, Three-dimensional physics-based registration of pelvic system using 2D dynamic magnetic resonance imaging slices, Strain 56 (2020) e12339.

[9] A. C. Ogier, S. Rapacchi, A. Le Troter, M.-E. Bellemare, 3D dynamic MRI for pelvis observation-a first step, in: 2019 IEEE 16th International Symposium on Biomedical Imaging (ISBI 2019), IEEE, pp. 1801-1804. 
[10] K. Makki, A. Bohi, A. C. Ogier, M.-E. Bellemare, A new geodesic-based feature for characterization of 3D shapes: application to soft tissue organ temporal deformations, 25th International Conference on Pattern Recognition (ICPR2020), Jan 2021, Milan, Italy (2020).

[11] J. Brüning, T. Hildebrandt, W. Heppt, N. Schmidt, H. Lamecker, A. Szengel, N. Amiridze, H. Ramm, M. Bindernagel, S. Zachow, et al., Characterization of the airflow within an average geometry of the healthy human nasal cavity, Scientific Reports $10(2020) 1-12$.

[12] X. Pennec, Advances in geometric statistics for manifold dimension reduction, in: Handbook of Variational Methods for Nonlinear Geometric Data, Springer, 2020, pp. 339-359.

[13] Q. Zheng, H. Delingette, K. Fung, S. E. Petersen, N. Ayache, Unsupervised shape and motion analysis of 3822 cardiac 4D MRIs of UK biobank, arXiv preprint arXiv:1902.05811 (2019).

[14] B. Abbas, J. Fishbaugh, C. Petchprapa, R. Lattanzi, G. Gerig, Analysis of the kinematic motion of the wrist from 4D magnetic resonance imaging, in: Medical Imaging 2019: Image Processing, volume 10949, International Society for Optics and Photonics, p. 109491E.

[15] S. Hong, J. Fishbaugh, G. Gerig, 4D continuous medial representation trajectory estimation for longitudinal shape analysis, in: International Workshop on Shape in Medical Imaging, Springer, pp. 125-136.

[16] K. Makki, B. Borotikar, M. Garetier, S. Brochard, D. B. Salem, F. Rousseau, In vivo ankle joint kinematics from dynamic magnetic resonance imaging using a registrationbased framework, Journal of biomechanics 86 (2019) 193-203.

[17] T. Heimann, H.-P. Meinzer, Statistical shape models for 3D medical image segmentation: a review, Medical image analysis 13 (2009) 543-563.

[18] X. Pennec, Statistical analysis of organs' shapes and deformations: the Riemannian and the affine settings in computational anatomy (2020).

[19] J. Fishbaugh, S. Durrleman, M. Prastawa, G. Gerig, Geodesic shape regression with multiple geometries and sparse parameters, Medical image analysis 39 (2017) 1-17.

[20] M. Zhang, P. Golland, Statistical shape analysis: From landmarks to diffeomorphisms, 2016.

[21] J. Fishbaugh, M. Prastawa, G. Gerig, S. Durrleman, Geodesic shape regression in the framework of currents, in: International Conference on Information Processing in Medical Imaging, Springer, pp. 718-729. 
[22] C. Abi Nader, N. Ayache, P. Robert, M. Lorenzi, A. D. N. Initiative, et al., Monotonic gaussian process for spatio-temporal disease progression modeling in brain imaging data, NeuroImage 205 (2020) 116266.

[23] R. Zolfaghari, N. Epain, C. T. Jin, A. Tew, J. Glaunes, A multiscale LDDMM template algorithm for studying ear shape variations, in: 2014 8th International Conference on Signal Processing and Communication Systems (ICSPCS), IEEE, pp. 1-6.

[24] A. Bône, O. Colliot, S. Durrleman, Learning distributions of shape trajectories from longitudinal datasets: a hierarchical model on a manifold of diffeomorphisms, in: Proceedings of the IEEE Conference on Computer Vision and Pattern Recognition, pp. 9271-9280.

[25] G. Peyré, L. D. Cohen, Geodesic methods for shape and surface processing, in: Advances in Computational Vision and Medical Image Processing, Springer, 2009, pp. 29-56.

[26] D. B. Malament, A remark about the "geodesic principle" in general relativity, in: Analysis and Interpretation in the Exact Sciences, Springer, 2012, pp. 245-252.

[27] Z. Sun, B. P. Lelieveldt, M. Staring, Fast linear geodesic shape regression using coupled logdemons registration, in: 2015 IEEE 12th International Symposium on Biomedical Imaging (ISBI), IEEE, pp. 1276-1279.

[28] H. Kim, M. Styner, J. Piven, G. Gerig, A framework to construct a longitudinal DWMRI infant atlas based on mixed effects modeling of dODF coefficients, arXiv preprint arXiv:2003.05091 (2020).

[29] R. Rios, R. De Crevoisier, J. D. Ospina, F. Commandeur, C. Lafond, A. Simon, P. Haigron, J. Espinosa, O. Acosta, Population model of bladder motion and deformation based on dominant eigenmodes and mixed-effects models in prostate cancer radiotherapy, Medical image analysis 38 (2017) 133-149.

[30] H. Luo, F. Jin, D. Yang, Y. Wang, C. Li, M. Guo, X. Ran, X. Liu, Q. Zhou, Y. Wu, Interfractional variation in bladder volume and its impact on cervical cancer radiotherapy: Clinical significance of portable bladder scanner, Medical physics 43 (2016) 4412-4419.

[31] D. G. Kendall, Shape manifolds, procrustean metrics, and complex projective spaces, Bulletin of the London mathematical society 16 (1984) 81-121.

[32] E. Nava-Yazdani, H.-C. Hege, C. von Tycowicz, A geodesic mixed effects model in Kendall's shape space, in: Multimodal Brain Image Analysis and Mathematical Foundations of Computational Anatomy, Springer, 2019, pp. 209-218.

[33] F. Billet, M. Sermesant, H. Delingette, N. Ayache, Cardiac motion recovery by coupling an electromechanical model and cine-MRI data: First steps, in: Proc. of the Workshop on Computational Biomechanics for Medicine III.(Workshop MICCAI-2008), volume 55, Citeseer, p. 176. 
[34] J. Lee, J. Woo, F. Xing, E. Z. Murano, M. Stone, J. L. Prince, Semi-automatic segmentation for 3D motion analysis of the tongue with dynamic MRI, Computerized Medical Imaging and Graphics 38 (2014) 714-724.

[35] K. Makki, B. Borotikar, M. Garetier, S. Brochard, D. B. Salem, F. Rousseau, Highresolution temporal reconstruction of ankle joint from dynamic MRI, in: 2018 IEEE 15th International Symposium on Biomedical Imaging (ISBI 2018), IEEE, pp. 12971300 .

[36] V. Arsigny, O. Commowick, N. Ayache, X. Pennec, A fast and log-euclidean polyaffine framework for locally linear registration, Journal of Mathematical Imaging and Vision 33 (2009) 222-238.

[37] M. Rahim, M.-E. Bellemare, R. Bulot, N. Pirró, A diffeomorphic mapping based characterization of temporal sequences: application to the pelvic organ dynamics assessment, Journal of mathematical imaging and vision 47 (2013) 151-164.

[38] T. Lewiner, H. Lopes, A. W. Vieira, G. Tavares, Efficient implementation of marching cubes' cases with topological guarantees, Journal of graphics tools 8 (2003) 1-15.

[39] W. Jakob, M. Tarini, D. Panozzo, O. Sorkine-Hornung, Instant field-aligned meshes., ACM Trans. Graph. 34 (2015) 189-1.

[40] M. F. Beg, M. I. Miller, A. Trouvé, L. Younes, Computing large deformation metric mappings via geodesic flows of diffeomorphisms, International journal of computer vision 61 (2005) 139-157.

[41] L. Váša, J. Rus, Dihedral angle mesh error: a fast perception correlated distortion measure for fixed connectivity triangle meshes, in: Computer Graphics Forum, volume 31, Wiley Online Library, pp. 1715-1724.

[42] E. W. Dijkstra, A note on two problems in connexion with graphs, Numerische mathematik 1 (1959) 269-271.

[43] J. A. Sethian, A fast marching level set method for monotonically advancing fronts, Proceedings of the National Academy of Sciences 93 (1996) 1591-1595.

[44] K. Crane, C. Weischedel, M. Wardetzky, The heat method for distance computation, Communications of the ACM 60 (2017) 90-99.

[45] A. J. Yezzi, J. L. Prince, An Eulerian PDE approach for computing tissue thickness, IEEE transactions on medical imaging 22 (2003) 1332-1339.

[46] O. Acosta, P. Bourgeat, M. A. Zuluaga, J. Fripp, O. Salvado, S. Ourselin, A. D. N. Initiative, et al., Automated voxel-based 3D cortical thickness measurement in a combined Lagrangian-Eulerian PDE approach using partial volume maps, Medical image analysis 13 (2009) 730-743. 
[47] N. Cedilnik, J. Duchateau, F. Sacher, P. Jaïs, H. Cochet, M. Sermesant, Fully automated electrophysiological model personalisation framework from CT imaging, in: International Conference on Functional Imaging and Modeling of the Heart, Springer, pp. 325-333.

[48] K. Crane, C. Weischedel, M. Wardetzky, Geodesics in heat: A new approach to computing distance based on heat flow, ACM Transactions on Graphics (TOG) 32 (2013) $1-11$.

[49] S. R. S. Varadhan, On the behavior of the fundamental solution of the heat equation with variable coefficients, Communications on Pure and Applied Mathematics 20 (1967) 431-455.

[50] G. Wang, X. Zhang, Q. Su, J. Shi, R. J. Caselli, Y. Wang, A. D. N. Initiative, et al., A novel cortical thickness estimation method based on volumetric Laplace-Beltrami operator and heat kernel, Medical image analysis 22 (2015) 1-20.

[51] A. Grigoryan, Heat kernel and analysis on manifolds, volume 47, American Mathematical Soc., 2009.

[52] R. J. LeVeque, Finite difference methods for ordinary and partial differential equations: steady-state and time-dependent problems, SIAM, 2007.

[53] W. Wessner, J. Cervenka, C. Heitzinger, A. Hossinger, S. Selberherr, Anisotropic mesh refinement for the simulation of three-dimensional semiconductor manufacturing processes, IEEE Transactions on Computer-Aided Design of Integrated Circuits and Systems 25 (2006) 2129-2139.

[54] S. G. Krantz, S. G. Krantz, Handbook of complex variables, Springer Science \& Business Media, 1999.

[55] R. E. Greene, S. G. Krantz, Function theory of one complex variable, volume 40, American Mathematical Soc., 2006.

[56] S. Durrleman, M. Prastawa, A. Routier, et. al., Deformetrica: learn from shapes, http: //www.deformetrica.org/, 2018.

[57] J. Lefèvre, G. Auzias, Spherical parameterization for genus zero surfaces using LaplaceBeltrami eigenfunctions, in: International Conference on Geometric Science of Information, Springer, pp. 121-129.

[58] A. Bohi, X. Wang, M. Harrach, M. Dinomais, F. Rousseau, J. Lefèvre, Global perturbation of initial geometry in a biomechanical model of cortical morphogenesis, in: 2019 41st Annual International Conference of the IEEE Engineering in Medicine and Biology Society (EMBC), IEEE, pp. 442-445. 
[59] B. T. Yeo, M. R. Sabuncu, T. Vercauteren, N. Ayache, B. Fischl, P. Golland, Spherical demons: fast diffeomorphic landmark-free surface registration, IEEE transactions on medical imaging 29 (2009) 650-668.

[60] S. Rusinkiewicz, Estimating curvatures and their derivatives on triangle meshes, in: Proceedings. 2nd International Symposium on 3D Data Processing, Visualization and Transmission, 2004. 3DPVT 2004., IEEE, pp. 486-493.

[61] N. Paragios, A level set approach for shape-driven segmentation and tracking of the left ventricle, IEEE transactions on medical imaging 22 (2003) 773-776.

[62] R. Goldman, Curvature formulas for implicit curves and surfaces, Computer Aided Geometric Design 22 (2005) 632-658.

[63] E. Albin, R. Knikker, S. Xin, C. O. Paschereit, Y. d'Angelo, Computational assessment of curvatures and principal directions of implicit surfaces from 3D scalar data, in: International Conference on Mathematical Methods for Curves and Surfaces, Springer, pp. 1-22.

[64] K. Makki, D. B. Salem, B. ben Amor, Towards the assessment of intrinsic geometry of implicit brain MRI manifolds, IEEE Access 9 (2021) 131054 - 131071.

[65] A. C. Ogier, M.-A. Hostin, M.-E. Bellemare, D. Bendahan, Overview of MR Image segmentation strategies in neuromuscular disorders, Frontiers in Neurology 12 (2021) 255.

[66] R. S. Hamilton, et al., Three-manifolds with positive Ricci curvature, J. Differential geom 17 (1982) 255-306.

[67] C. E. Weatherburn, Differential geometry of three dimensions, volume 1, Cambridge University Press, 2016.

[68] C. Avenel, E. Mémin, P. Pérez, Stochastic level set dynamics to track closed curves through image data, Journal of mathematical imaging and vision 49 (2014) 296-316.

\section{Appendices}

\section{A. Riemannian manifolds and geodesic flows}

In Section 3.2.2, we have demonstrated the geodesic nature of the obtained geometric flows from a numerical point of view. In this Appendix, we will try to establish an analogy with the classical geodesic flows in differential geometry for parametric surfaces. First we shall give an overview of the mathematical background of geodesics on Riemannian manifolds with an aim to help further explain the geodesic nature of the obtained curves and lengths. A 2-manifold is just a surface in three dimensional space. In differential geometry, manifolds 
are "topological spaces" which locally resemble Euclidean. $(\mathcal{M}, g)$ is a called Riemannian manifold equipped with a metric $g$ if it is a differential manifold where the metric satisfies:

$$
g \in \operatorname{Sym}_{++}^{2}\left(\mathcal{T}_{x} \mathcal{M}\right), \quad \forall \quad x \in \mathcal{M}
$$

where $\operatorname{Sym}_{++}^{2}$ is the group of symmetric positive definite bilinear forms defined on the tangent space.

While the Euclidean metric is the conventional Euclidean distance (i.e. the distance of the straight line between two points), the Riemannian metric controlling intrinsic properties of a curved surface allows one to define distances and angles on the manifold, that best preserves the Euclidean geometry of the local tangent space.

Let $u$ and $v$ be two vectors of the tangent plane $\mathcal{T}_{x_{0}} \mathcal{M}$ with origin $x_{0}$, then the Riemann metric can be defined in the sense of inner (scalar) product by: $g\left(t, x_{0}, u, v\right)=\|u\| \cdot\|v\| \cdot \cos (u, v)$ with $t \in[0,1]$.

In Riemannian geometry, a curve with tangential acceleration zero is called a geodesic. Geodesics are also characterized by the property of having vanishing intrinsic curvature. The shortest geodesic between two surface points $x_{0}$ and $x_{1}$ can be obtained by minimizing the following Dirichlet energy, subject to fixed endpoints $\gamma(0)=x_{0}$ and $\gamma(1)=x_{1}$ :

$$
E_{D}(\gamma)=\frac{1}{2} \int_{0}^{1}\left|\gamma^{\prime}(t)\right|^{2} d t=\frac{1}{2} \int_{0}^{1} g_{\gamma(t)}\left(\gamma^{\prime}(t), \gamma^{\prime}(t)\right) d t
$$

The length of the shortest geodesic curve $\gamma$ is called a geodesic distance, it is like a flexibility when we define distance:

$$
L(\gamma)=\int_{0}^{1} \sqrt{g_{\gamma(t)}\left(\gamma^{\prime}(t), \gamma^{\prime}(t)\right)} d t
$$

If the metric $g$ reduces to the Kronecker delta $\delta_{i j}$, then the Eq. 24 reduces to:

$$
L(\gamma)=\int_{0}^{1}\left\|\gamma^{\prime}(t)\right\| d t
$$

Fig. 14 illustrates the Riemannian geodesic distances between the point $x_{0} \in \mathcal{M}$ and each surface point $x_{1} \in \mathcal{M}$ (example for one reconstructed bladder surface).

In Riemannian geometry, each spherical mapping method is subsumed by Ricci flow (the generic model). Like the heat equation developed by Fourier $\frac{\partial h}{\partial t}-\alpha \Delta h=0$, where $\alpha$ is the diffusivity constant, the Ricci flow is a non-linear PDE, but resulting from geometry rather than physics [66]. The Ricci flow is therefore a way of changing the metric tensor over time until it converges toward a positive constant (the sphere curvature), according to the parabolic PDE $\frac{\partial g}{\partial t}+2 R i c_{g}=0$. Like the Riemannian metric $g$, the Ricci curvature tensor $R i c_{g}$ is a symmetric bilinear form defined as the trace of the curvature tensor. It is therefore a mean of the information contained in the Riemann curvature tensor.

With a separation of space and time variables, the above defined heat equation (parabolic PDE) can be splitted into two elliptic PDEs that can be easily solved separately. In this 
particular case, the separation of variables is a result of the spectral theorem. More details are provided in Appendix C.

In the same analogy, and assuming that the solution is becoming time-independent under the imposed constraints, the Dirichlet energy to be minimized is the one given in Eq. 14 . The harmonic map $h$ minimizing this energy is the optimal solution of an elliptic PDE. By definition, a map $h$ is called harmonic if its Laplacian vanishes, $\Delta h=0$. We then propose to recover the curve-shortening flow by integrating the normalized gradient of $h$.

The resulting geometric flow is gradient like, i.e. a process that modify the surface curve toward the sphere by moving its points perpendicularly to the curve (the tangential acceleration vanishes), at a speed proportional to the curvature. The mean curvature (extrinsic)

of the surface $\mathcal{M}$ can be derived from the obtained harmonic interpolant according to the divergence formula: $\kappa(x)=\frac{1}{2} \operatorname{div}\left(\frac{\nabla h(x)}{\|\nabla h(x)\|}\right), \forall x \in \mathcal{M}$. A visual example is provided in Fig 15.

Replacing $\gamma^{\prime}$ with $N=\frac{\nabla h}{\|\nabla h\|}$ in Eq. 25, and assuming again that the solution is timeindependent, then the geodesic lengths/distances can be defined (in the sense of optimization) by:

$$
L^{*}\left(\Phi_{N}\right)=\min _{L}\left(\int_{\partial_{1} \Omega}^{\partial_{0} \Omega} \| \nabla L-\left.N\right|^{2} d x\right)
$$

The numerical integration of the above equation over $\Omega$ is performed using an iterative Gauss-Seidel relaxation method, for locally solving the following PDE:

$$
\nabla L \cdot N=\|\nabla L\| \cdot\|N\| \cdot \cos (\nabla L, N)=1
$$

where the left term $(\|\nabla L\| .\|N\| \cdot \cos (\nabla L, N))$ is the scalar product between the two vectors, while the right term (1) controls the distances and angles: by forcing infinitesimal lengths to be unitary and by specifying the direction (angle=0) in such a way that the gradient of the scalar distance function $\nabla L$ coincides with the velocity vector field $N$. Thus, the integrated trajectory is qualified as a geodesic and its length is locally defined and shortest in the sense of scalar product.

\section{B. Normalized gradient vector field}

To prove that the computed correspondence trajectories are geodesics, it is mandatory to prove that $N$ is conservative. For this, it is sufficient to prove that $N$ is curl free (irrotational) in the vicinity of each heat isosurface. But, this is non-trivial, since $\|\nabla h\| \neq c s t e$ generally so that:

$\operatorname{curl}(N)=\nabla \times N=\nabla \times\left(\frac{\nabla h}{\|\nabla h\|}\right)=\nabla\left(\frac{1}{\|\nabla h\|}\right) \times \nabla h+\frac{1}{\|\nabla h\|} \underbrace{\nabla \times \nabla h}_{=\operatorname{curl}(\nabla h)=0 .}=\frac{1}{\|\nabla h\|^{2}} \nabla(\|\nabla h\|) \times \nabla h$.

Overall $\Omega$, for each isosurface $h=h_{\text {iso }}$, the fact that $\operatorname{curl}(N)=0$ depends essentially on how the vector field $N$ is defined away from that isosurface. However, on each isosurface, $N$ is 
well defined and conservative [67].

- Formulation 1: Suppose $N$ to be the normalized gradient of $h$ over $\Omega$. In this case, $\nabla \times N=0$, when evaluated on the isosurface $h_{\text {iso }}$ if and only if $\|\nabla h\|=$ cste.

- Formulation 2: Define the function $f=\frac{1}{\|\nabla h\|}\left(h-h_{i s o}\right)$ inside $\Omega$. Suppose now that the surface we are interested in is $\Gamma=\{f=0\}$. Developing the expression of the gradient of $f$, we obtain:

$$
\nabla f=\frac{\nabla h}{\|\nabla h\|}-\frac{\left(h-h_{i s o}\right) \nabla h \cdot \nabla^{2} h}{\|\nabla h\|^{3}},
$$

where $\nabla^{2}($.$) is the Hessian operator. It is clear that the second term of the right-hand$ side of the Eq. (28) vanishes on $\Gamma$ (since $h=h_{i s o}$ ). Consequently, $\nabla f$ restricted to the surface is still the unit normal vector field $N$. Moreover, $\nabla \times \nabla f$ is clearly zero as argued before.

More generally, for a compact smooth surface $\Gamma \subset \mathbb{R}^{n}$, there exists a real $r>0$ such that on the set $\omega=\left\{x \in \mathbb{R}^{3} \quad \mid \operatorname{dist}(x, \Gamma)<r\right\}$ one can solve the Eikonal PDE $|\nabla f|=1$ to get a continuous function $f: \omega \mapsto \mathbb{R}$ which satisfies $\Gamma=f^{-1}(0)$ and such that $\nabla f$ is the unit normal vector field for any level set $f^{-1}(c)$. Under this formulation, the unit normal vector field $N=\nabla f$ is curl-free in a narrowband $\omega$ of the surface $\Gamma$. The real number $r$ is related to the radius of the surface mean curvature. Fig. 16.(c) illustrates that for each set $\omega_{c} \subset \Omega$ and for a small radius $r$, we have $|\nabla h|=$ cste.

\section{Background on harmonic analysis for 2-manifolds}

The harmonic analysis on Riemannian manifolds is in fact a generalization of Fourier spectral shape analysis. For which, the Fourier modes on $[0,1]$ are solutions of $f^{\prime \prime}=-\lambda f$ with boundary conditions (Dirichlet, Neumann, etc.).

Again, the more general heat equation is the principal ingredient. This equation reads:

$$
\frac{\partial h}{\partial t}=\alpha \Delta h
$$

where the function $h$ describes the temperature distribution over the manifold.

In the homogeneous case, the boundary conditions satisfy $h\left(\partial_{x_{0}} \mathcal{M}\right)=h\left(\partial_{x_{L}} \mathcal{M}\right)=0$. Looking now for a solution which is not identically zero satisfying the boundary conditions and for which the dependence of $h$ on $x, t$ is separated, where $x \in \mathcal{M}$ and $t$ denotes the time, that is:

$$
h(x, t)=f(x) \cdot T(t) .
$$

Using the product rule and substituting $h$ back into equation 29 , we obtain:

$$
\frac{T^{\prime}(t)}{\alpha T(t)}=\frac{\Delta f(x)}{f(x)}
$$


Since the left hand side depends only on $t$ and the right hand side only on $x$, then both sides are equal to some constant value $-\lambda$. Thus:

$$
T^{\prime}(t)=-\alpha \lambda T(t),
$$

and

$$
\Delta f(x)=-\lambda f(x)
$$

where: $-\lambda$ represent the eigenvalues for both differential operators, and $T(t)$ and $f(x)$ are corresponding eigenfunctions. If we only consider the heat vibration modes in the spatial domain, then the heat equation reduces to Eq. 33 (a special case of the Poisson equation $\Delta \phi=f$, with $f=\lambda \phi$ ), for which, the solutions become time-independent.

\section{Definition:}

Given a complete 2-Riemannian manifold without boundary (closed surface), $(\mathcal{M}, g)$ equipped with a local metric tensor $g$ and with a local coordinate system $u: x \in \mathcal{M} \rightarrow \mathbb{R}^{2}$ / $u(x)=\left(u_{1}, u_{2}\right)$, the LBO acting on $C^{\infty}$ functions is defined by:

$$
\Delta_{\mathcal{M}} f(u)=\frac{1}{\sqrt{\operatorname{det}(g)}} \sum_{i, j} \partial_{u_{j}}\left(\sqrt{\operatorname{det}(g)} g^{i, j} \partial_{u_{i}} f(u)\right)
$$

Consider now the Dirichlet eigenvalue problem:

$$
\left\{\begin{array}{l}
\Delta f=-\lambda f \quad \text { in } \quad \Omega, \\
f=0 \text { on } \partial \Omega .
\end{array}\right.
$$

where: $\Omega \subset \mathcal{M}$. Solving this problem gives an infinite set of sorted eigenvalues $0 \leq \lambda_{1} \leq$ $\lambda_{2} \leq \lambda_{3} \ldots$ and a set of corresponding eigenfunctions $\Phi_{1}, \Phi_{2}, \Phi_{3} \ldots$ (orthogonal in the sense of the scalar product), which form together an orthonormal basis of $L^{2}(\mathcal{M})$. An example showing the five first eigenfunctions for a bladder surface is given in Fig. 17.

As proposed in [57], the principle of spherical mapping is as follows: for a surface point $x \in \mathcal{M}$, there exists a $C^{\infty}$ diffeomorphism $\Phi$, satisfying:

$$
\begin{gathered}
\Phi: \mathcal{M} \rightarrow \mathbb{S}^{2} \\
x \longmapsto \frac{\left(\Phi_{1}(x), \Phi_{2}(x), \Phi_{3}(x)\right)}{\sqrt{\Phi_{1}(x)^{2}+\Phi_{2}(x)^{2}+\Phi_{3}(x)^{2}}}
\end{gathered}
$$

By solving Laplace equation under the condition of the equilibrium state, one can redefine 
the above defined diffeomorphic spherical mapping with the following application:

$$
\begin{gathered}
\Phi_{n}: \mathcal{M} \rightarrow \mathbb{S}^{2} \\
x \longmapsto \frac{\Phi_{N}(x)}{\left\|\Phi_{N}(x)\right\|}=\frac{\left(\Phi_{N_{i}}(x), \Phi_{N_{j}}(x), \Phi_{N_{k}}(x)\right)}{\sqrt{\Phi_{N_{i}}(x)^{2}+\Phi_{N_{j}}(x)^{2}+\Phi_{N_{k}}(x)^{2}}},
\end{gathered}
$$

such that $\Phi_{N}\left(x_{0}\right)=\left(x_{0}-c_{0}\right)+\int_{x_{0}}^{\partial_{0} \Omega} N(x) d x$, where $c_{0}$ is the center of the surrounding sphere, and $x_{0} \in \mathcal{M}$. Intuitively, integrating the normal velocity vector field $v=N$ (defined in Section 3.2.2) gives a smooth bijective map $\Phi_{N}$ which transforms each surface point $x_{0} \in \mathcal{M}$ into its unique corresponding point in the surrounding sphere $x_{1}$, modulo translation (see examples of Fig. 18). The way in which surface points move along the curve normal directions within the Eulerian Framework resembles the principle used in level set methods to track evolving curves while providing a notion of correspondence [68]. 

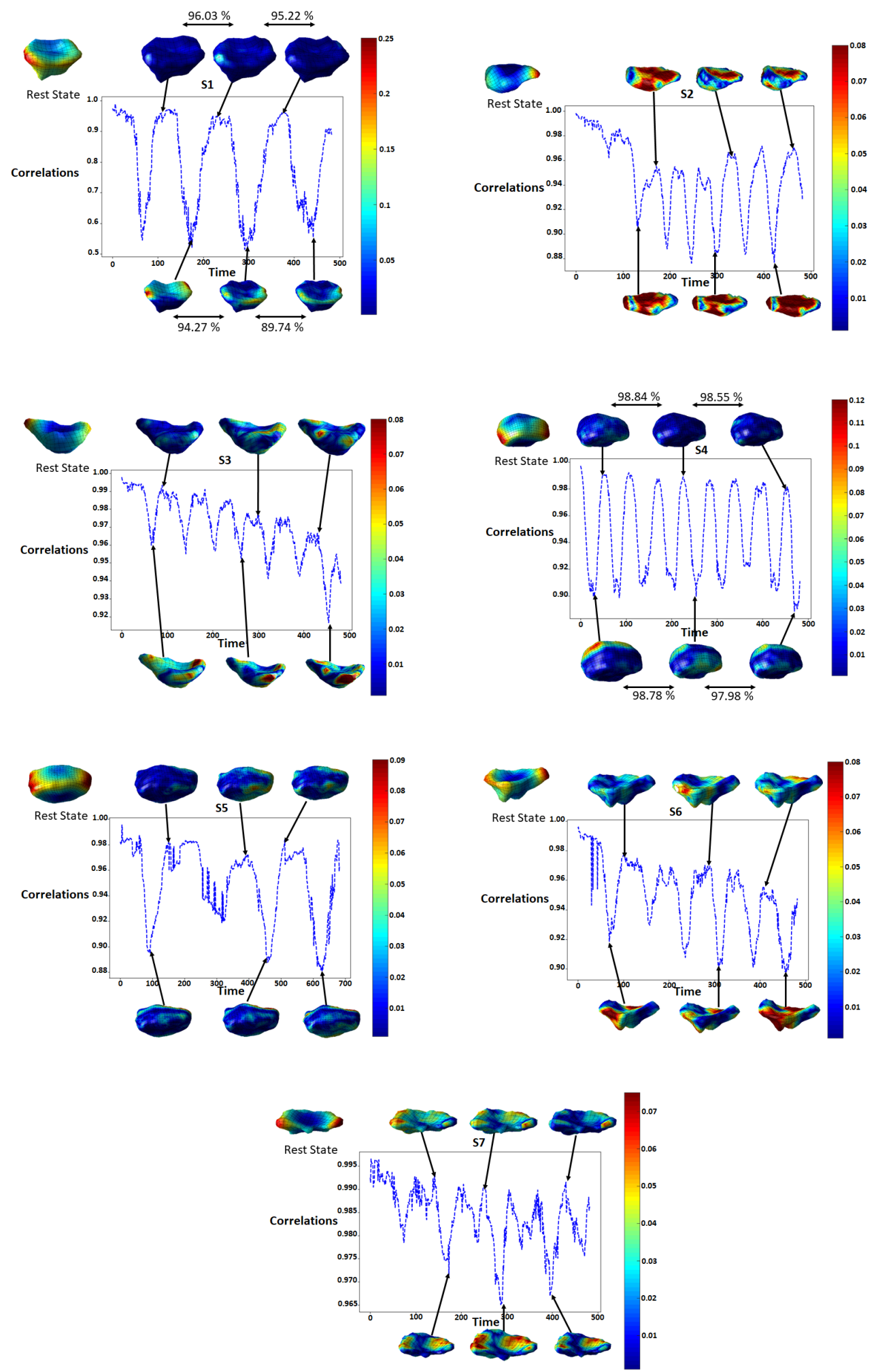

Figure 9: Characterization of the different loading phases of the bladder using the proposed feature. Each subfigure corresponds to one subject. For each subject, we show the cyclical behavior in surface motion patterns: at maximum of expiration states (top meshes), and at maximum of inspiration states (buttom meshes). Colormaps depict feature changes in $\mathrm{mm}^{-1}$ and range from blue (no deformation) to red (high deformation). 


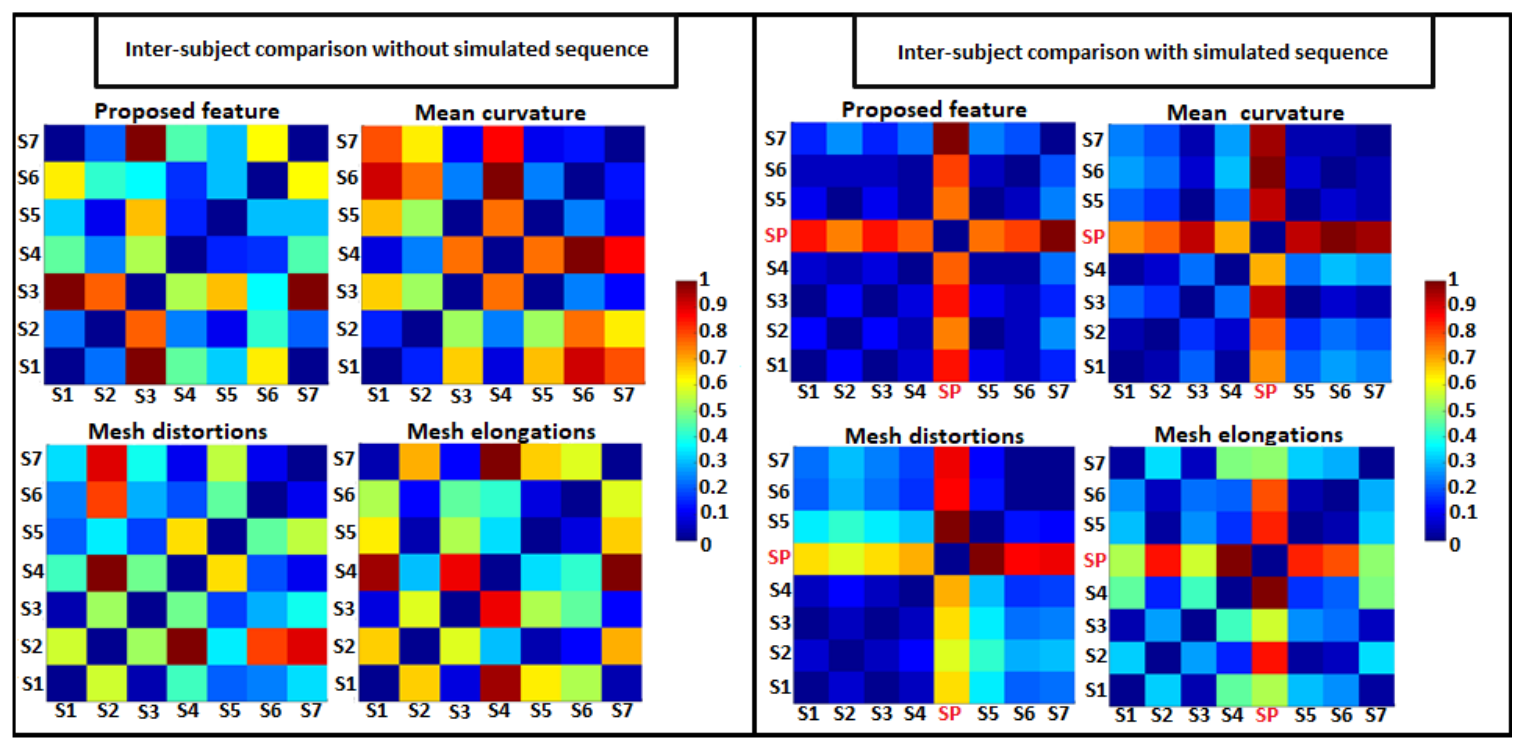

Figure 10: Inter-subject comparison: normalized distance matrices between maximal temporal deformations (i.e. deformation depths). The left panel depicts the results for the volunteer-only comparisons. The right panel shows the comparisons after introducing the artificial sequence into the mix. 


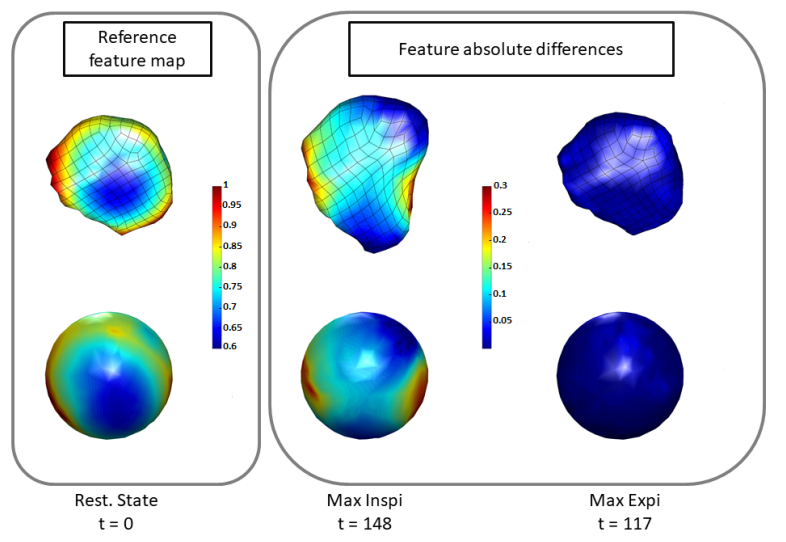

(a) Proposed feature (in $\mathrm{mm}^{-1}$ )

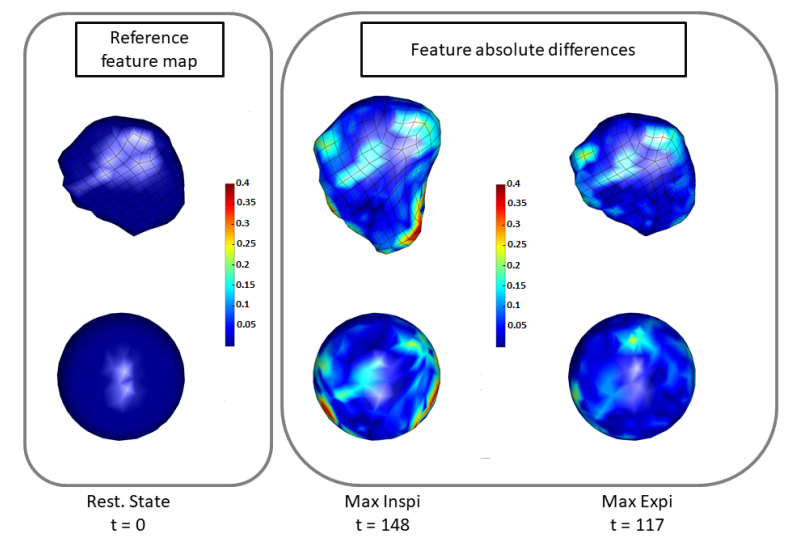

(c) Mesh distortions (in degrees)

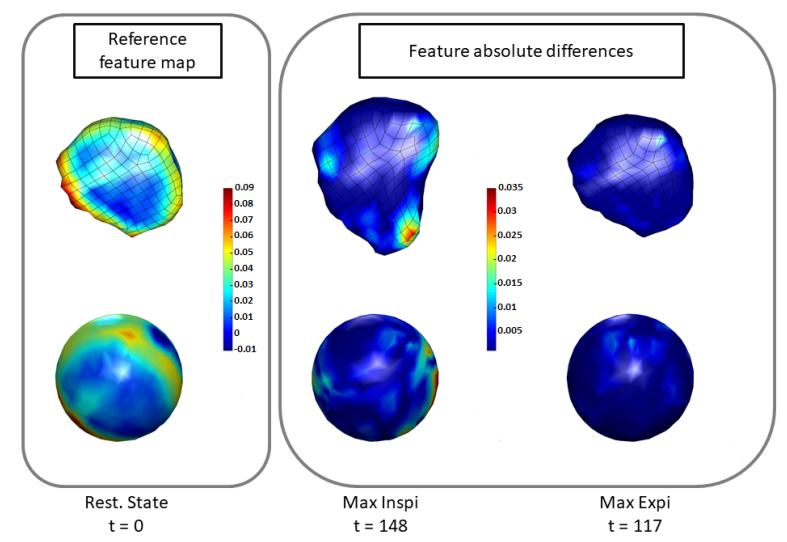

(b) Mean curvature (in $\mathrm{mm}^{-1}$ )

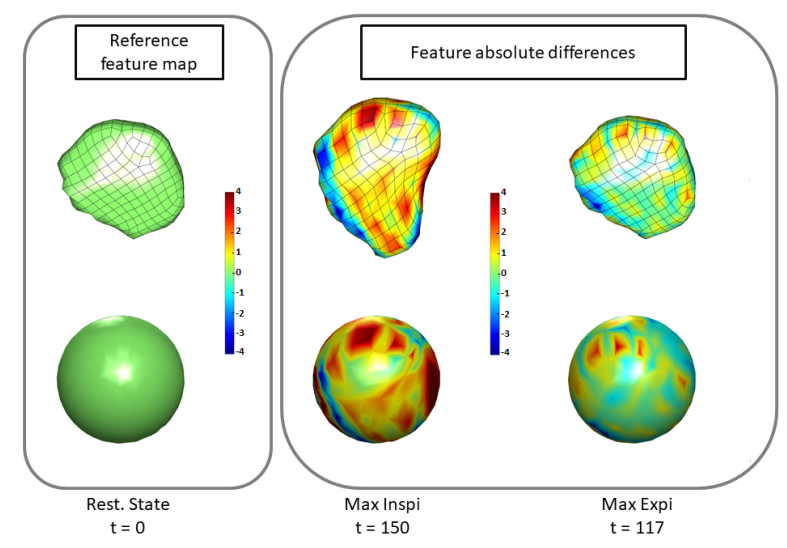

(d) Mesh elongations (in $\mathrm{mm}$ )

Figure 11: Feature projection on the sphere. For each panel, the first column represents the reference feature map, while the $2^{\text {nd }}$ and $3^{\text {rd }}$ columns represent the feature changes at maximal range of motion w.r.t the reference state (patterns of the highest simulated organ deformation). 


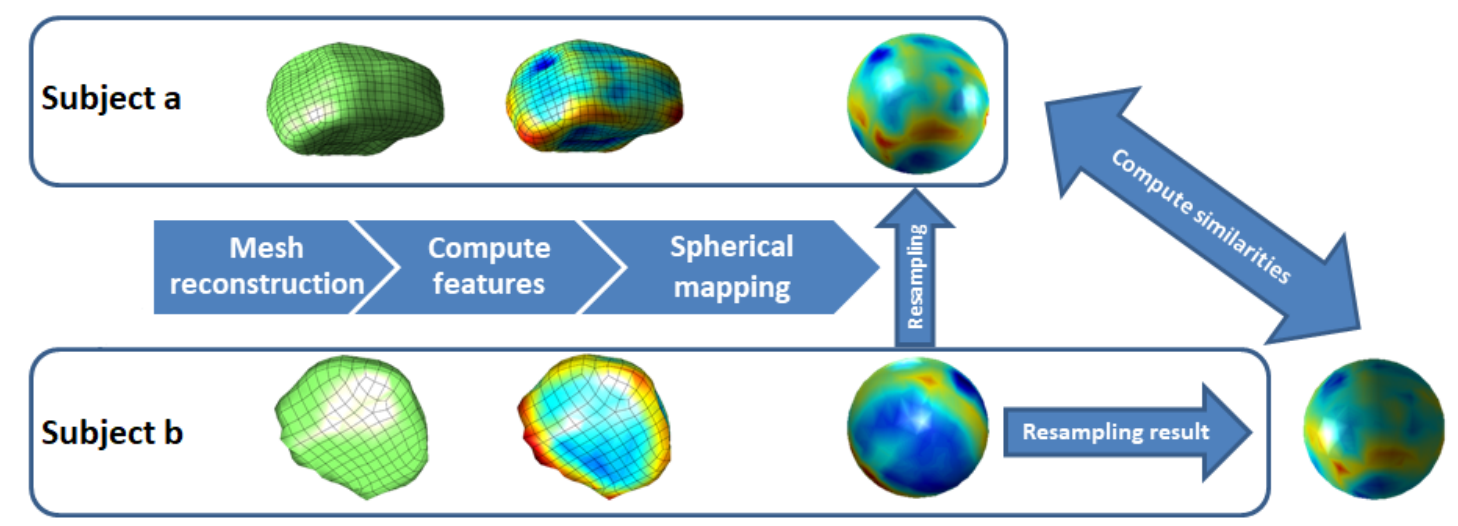

Figure 12: The pipeline of the proposed framework of comparing two bladder surfaces with different parameterizations. This Figure summarizes the key steps leading to perform inter-subject comparisons. In this example, comparisons are based on the proposed feature. To complete this process, we resample the projected feature texture for "subject b" to fit the feature map projection for "subject a" using a KdTree interpolation.
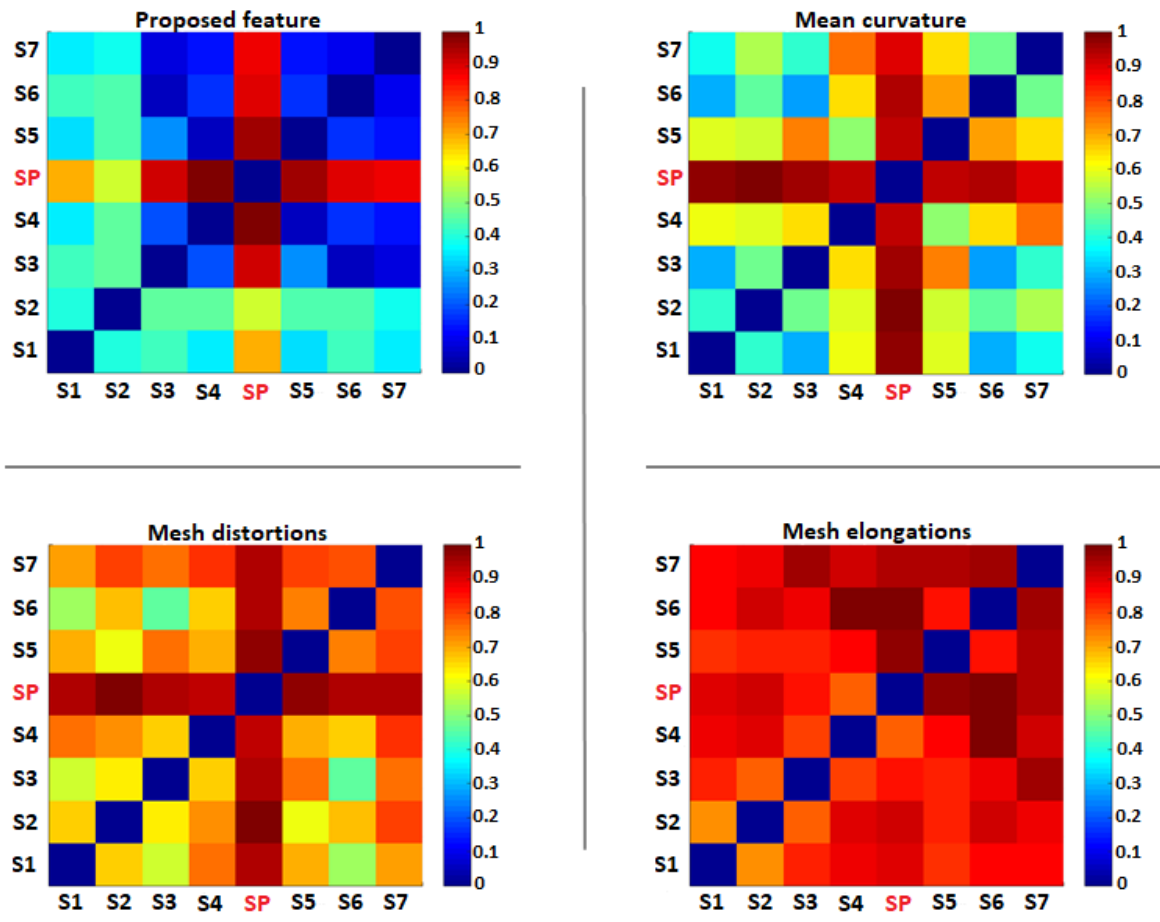

Figure 13: Inter-subject comparison using LBO spherical mapping with a Kdtree interpolation. Comparisons were based on the correlations between subjects' motion patterns in a common shape space (the unit sphere): For each feature, the matrix elements were filled using the distance function $\operatorname{dist}\left(\overline{\mathcal{F}}_{i}, \overline{\mathcal{F}}_{j}\right)=$ $1-\operatorname{norm} \operatorname{corr}\left(\overline{\mathcal{F}}_{i}, \overline{\mathcal{F}}_{j}\right)$, where $\overline{\mathcal{F}}_{i}$ is the resampled average map for distances between temporal feature vectors and the reference one, for subject $S_{i}$, and normcorr is the normalized correlation function. 


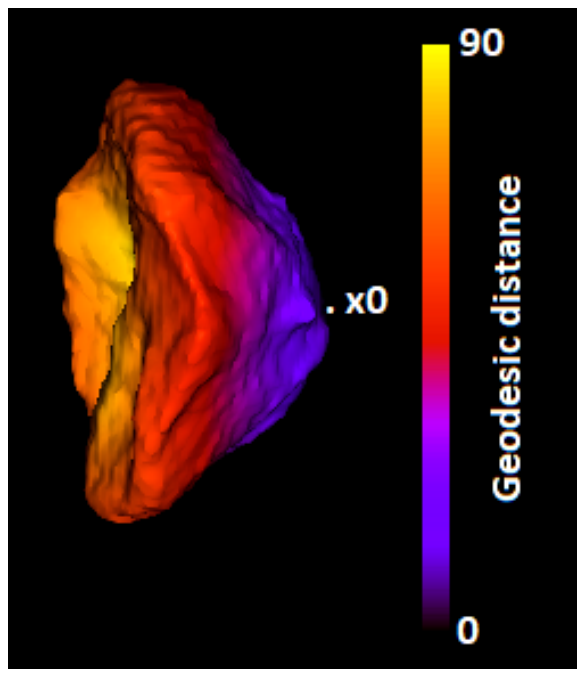

Figure 14: Geodesic distances on Riemannian manifolds (expressed in mm for the bladder example): the lengths of the shortest paths connecting each vertex to the vertex $x_{0}$, along the manifold.
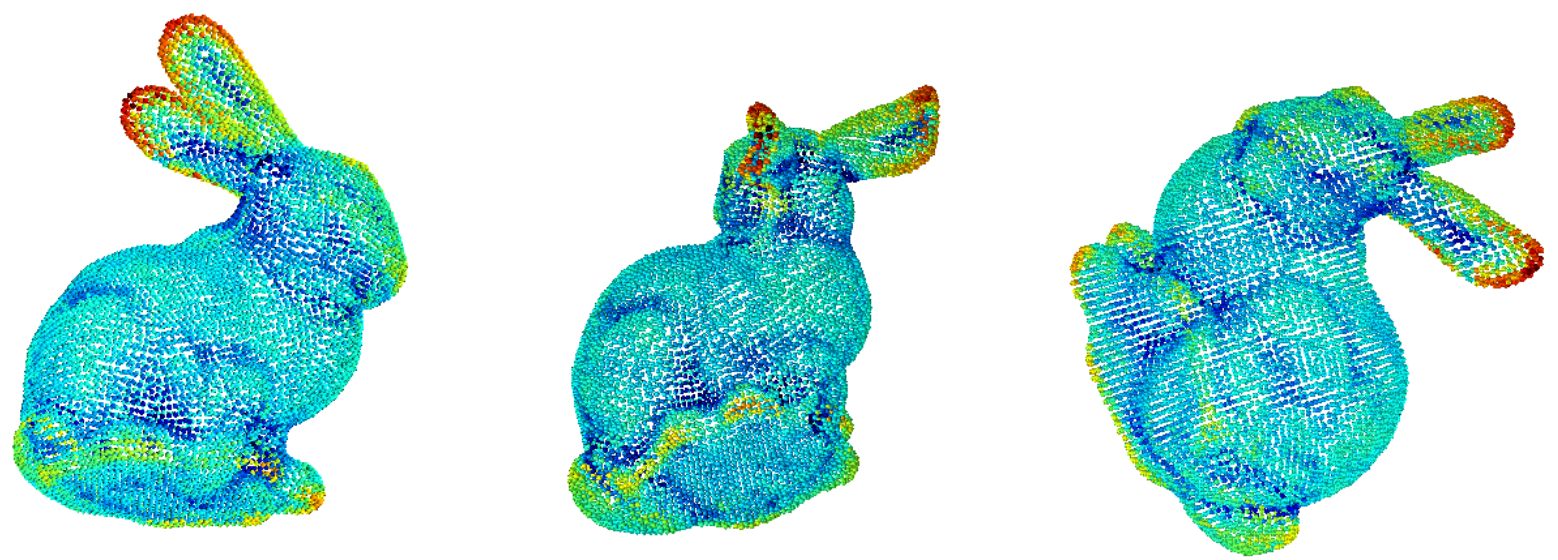

Figure 15: Obtained mean curvature for the isosurface $h=0.98$. 


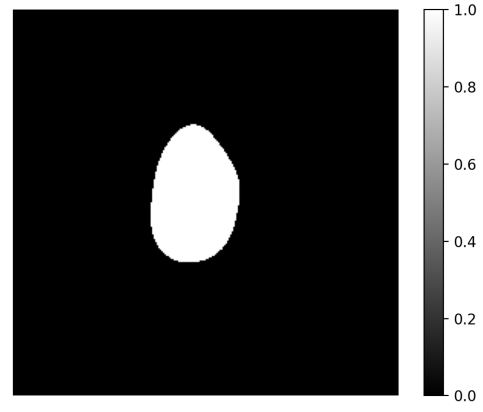

(a) Input shape

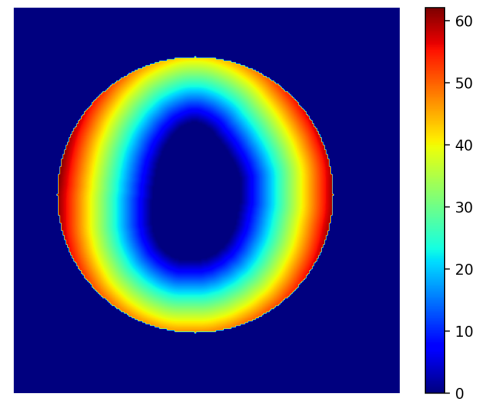

(d) Fast marching distance $L_{F M}$

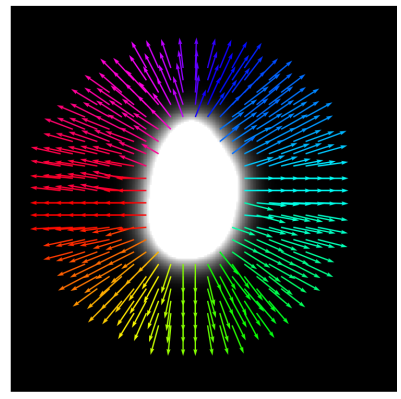

(g) $\nabla L_{F M}$

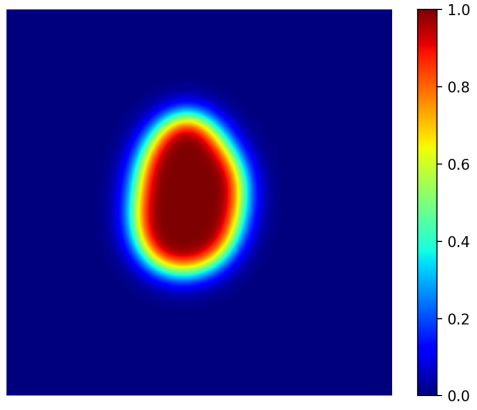

(b) $h$

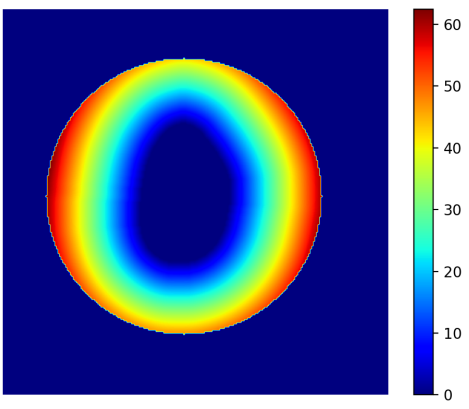

(e) Our distance $L$

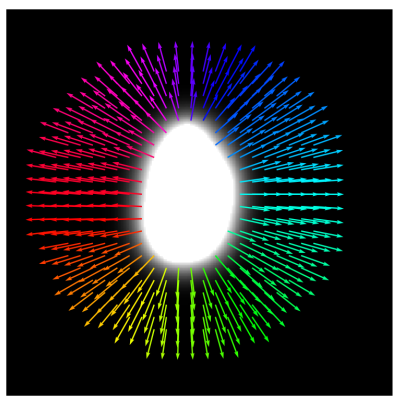

(h) $N$

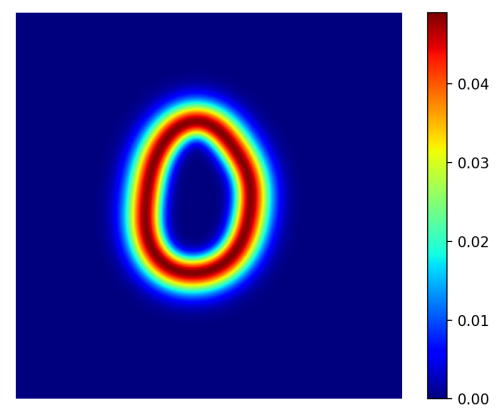

(c) $\|\nabla h\|_{2}$

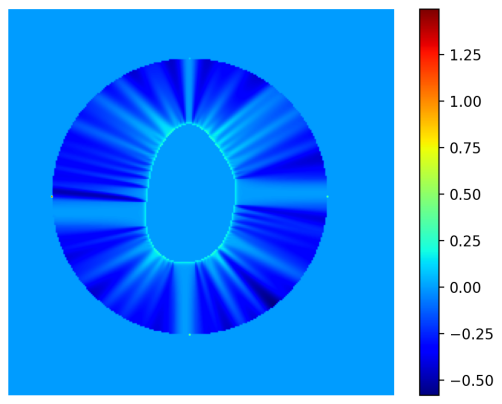

(f) $L-L_{F M}$

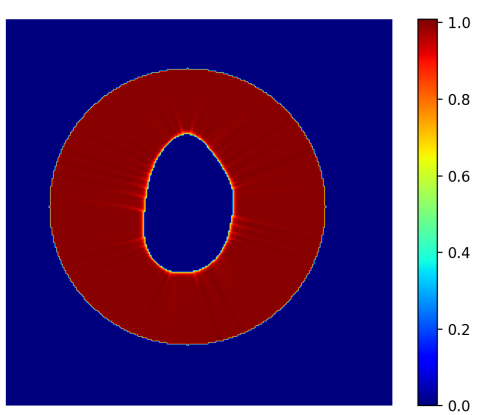

(i) Scalar product: $\nabla L_{F M} \cdot N$

Figure 16: Method comparison with the Fast marching method. Comparison is based on the scalar product of their gradient vector fields. 


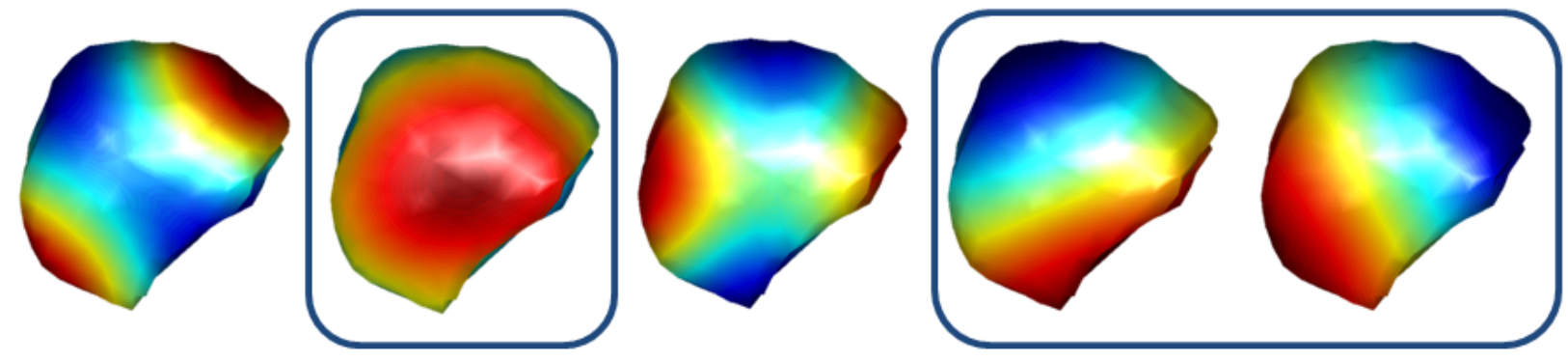

Figure 17: Surface spectral analysis: five first non-trivial LBO eigenfunctions (sorted from left to right). Colormap goes from blue (negative) to red (positive). The eigenfunctions with only two nodal domains $\Phi_{2}$, $\Phi_{4}$, and $\Phi_{5}$ were used for spherical parameterization.
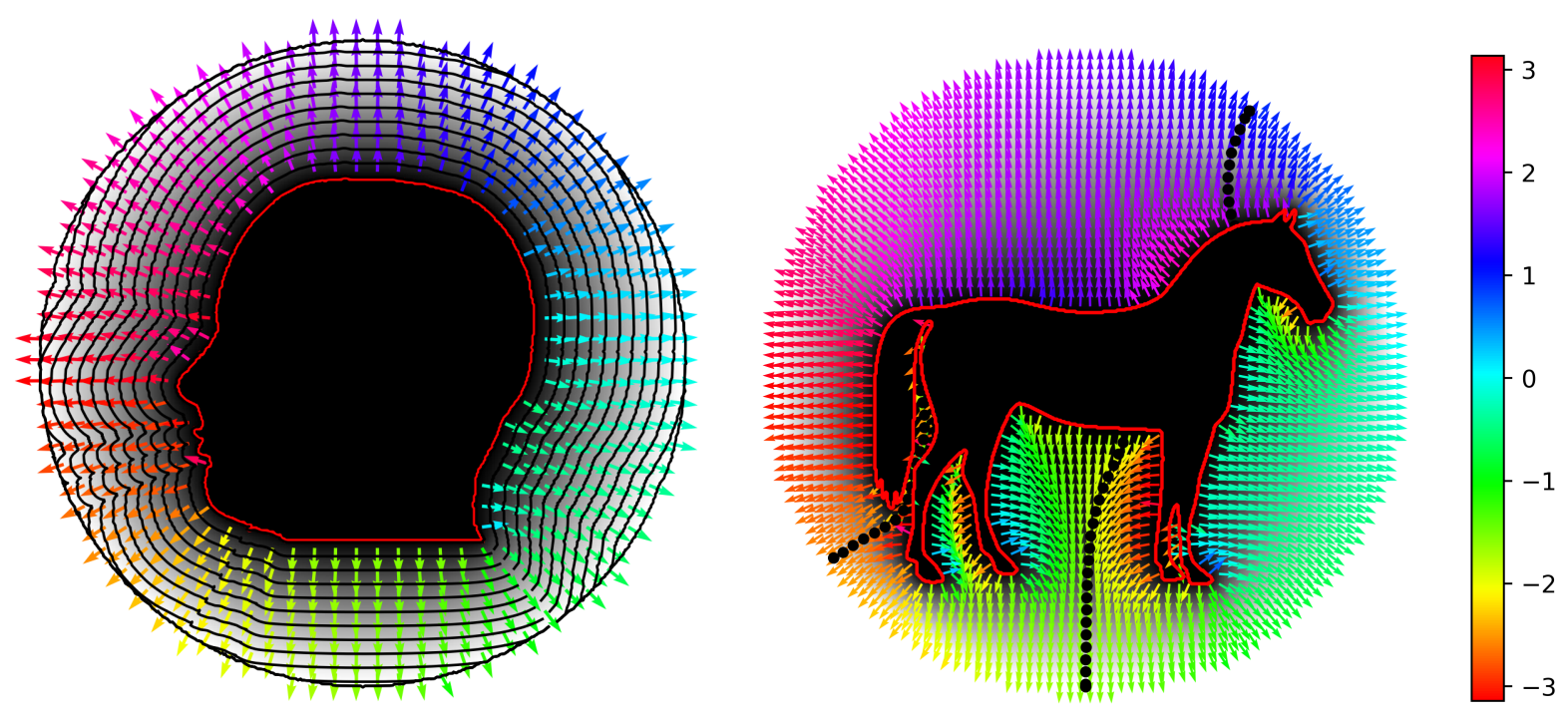

Figure 18: Numerical integration of the velocity vector field (2D examples). Left: the dark curves indicate the trajectory of shape contour,or equivalently, the isocurves of the distance function. Right: the entire trajectories of some contour points, where the colorbar encodes the direction of the gradient vector field $N$ in radians. Note that the principle remains the same in $3 \mathrm{D}$. 